\title{
MeV neutrino dark matter: relic density, lepton flavour violation and electron recoil
}

\author{
Juri Fiaschi, ${ }^{a}$ Michael Klasen, ${ }^{a}$ Miguel Vargas, ${ }^{b}$ Christian Weinheimer ${ }^{b}$ and \\ Sybrand Zeinstra ${ }^{a}$ \\ ${ }^{a}$ Institut für Theoretische Physik, Westfälische Wilhelms-Universität Münster, \\ Wilhelm-Klemm-Straße 9, 48149 Münster, Germany \\ ${ }^{b}$ Institut für Kernphysik, Westfälische Wilhelms-Universität Münster, \\ Wilhelm-Klemm-Straße 9, 48149 Münster, Germany \\ E-mail: fiaschi@uni-muenster.de, michael.klasen@uni-muenster.de, \\ m_varg03@uni-muenster.de, weinheimer@uni-muenster.de, \\ swzeinstra@uni-muenster.de
}

AbStRaCt: Right-handed neutrinos with $\mathrm{MeV}$ to $\mathrm{GeV}$ mass are very promising candidates for dark matter (DM). Not only can they solve the missing satellite puzzle, the cuspcore problem of inner DM density profiles, and the too-big-to fail problem, i.e. that the unobserved satellites are too big to not have visible stars, but they can also account for the Standard Model (SM) neutrino masses at one loop. We perform a comprehensive study of the right-handed neutrino parameter space and impose the correct observed relic density and SM neutrino mass differences and mixings. We find that the DM masses are in agreement with bounds from big-bang nucleosynthesis, but that these constraints induce sizeable DM couplings to the charged SM leptons. We then point out that previously overlooked limits from current and future lepton flavour violation experiments such as MEG and SINDRUM heavily constrain the allowed parameter space. Since the DM is leptophilic, we also investigate electron recoil as a possible direct detection signal, in particular in the XENON1T experiment. We find that despite the large coupling and low backgrounds, the energy thresholds are still too high and the predicted cross sections too low due to the heavy charged mediator, whose mass is constrained by LEP limits.

Keywords: Beyond Standard Model, Cosmology of Theories beyond the SM, Neutrino Physics

ARXiv EPRINT: 1908.09882 


\section{Contents}

1 Introduction 1

2 The SLIM model with MeV neutrino DM 3

2.1 Particle content 3

2.2 Lagrangian 3

2.3 Scalar masses and mixings after electroweak symmetry breaking 4

3 Collider, cosmological and neutrino constraints 4

3.1 Collider constraints 5

$\begin{array}{lll}3.2 & \text { Cosmological constraints } & 6\end{array}$

$\begin{array}{lll}3.3 & \text { Neutrino constraints } & 7\end{array}$

4 Right-handed neutrino DM relic density, mass and lepton couplings $\quad 8$

5 Lepton flavour violation and experimental constraints 10

6 Electron recoil cross section and experimental sensitivity 12

$\begin{array}{lll}6.1 & \text { Theoretical expectations } & 14\end{array}$

$\begin{array}{lll}6.2 & \text { Experimental sensitivity } & 15\end{array}$

$\begin{array}{llr}7 & \text { Conclusion } & 18\end{array}$

\section{Introduction}

While there is overwhelming evidence from many different length scales for the existence of dark matter (DM) in the Universe, its nature is still an unsolved problem in astroparticle physics [1]. Weakly Interacting Massive Particles (WIMPs) are very promising candidates, since - for masses in the $\mathrm{GeV}$ to $\mathrm{TeV}$ range and electroweak coupling strength - they lead in a straightforward way to the correct DM relic density. The latter has now been measured by Planck with the very high accuracy $\Omega h^{2}=0.1186 \pm 0.0020$, where $h$ denotes the present Hubble expansion rate $H_{0}$ in units of $100 \mathrm{~km} \mathrm{~s}^{-1} \mathrm{Mpc}^{-1}$ [2].

For many years, the lightest supersymmetric (SUSY) particle, the lightest neutralino, stabilised by a discrete $R$-parity symmetry, has been favoured, since there exist many other theoretical motivations for SUSY [3]. The neutralino relic density [4-19], direct detection [20] and LHC production cross sections [21-27] can even be computed with a precision that rivals the experimental one. However, neither the leading direct detection experiment in this mass range, XENON1T [28], nor the LHC [29] have so far produced any evidence for SUSY DM. In contrast, the discovery of a scalar boson of mass $125 \mathrm{GeV}$, consistent with the properties of the Standard Model (SM) Higgs boson, by ATLAS [30] 
and CMS [31] may imply the existence of other scalars in Nature. When stabilised by a discrete $Z_{2}$ symmetry similar to $R$-parity, the lightest neutral eigenstate may well be the DM particle, as is the case e.g. in the inert doublet model [32-34].

In addition to the evidence for DM, the observed solar and atmospheric neutrino mass differences of $(7.59 \pm 0.20) \cdot 10^{-5} \mathrm{eV}^{2}$ and $(2.43 \pm 0.13) \cdot 10^{-3} \mathrm{eV}^{2}[35]$ require physics beyond the SM. Adding right-handed neutrinos to the SM has the advantage that they do not only allow for neutrino mass generation through different variants of the seesaw mechanism, but that they also help to restore parity at higher energy. If the seesaw mechanism is furthermore realised at one loop, DM and neutrino masses become closely related as e.g. in the scotogenic model [36] or variants thereof [37-41]. ${ }^{1}$ The detection prospects of DM depend not only on its spin and other quantum numbers, but also strongly on its mass. While the observed large scale structure of the Universe has long been believed to favour the $\mathrm{GeV}$ to $\mathrm{TeV}$ cold DM regime [42], warm DM, and in particular keV sterile neutrinos, seem to account better for missing satellite galaxies, the cusp-core problem of inner DM density profiles and the too-big-to fail problem, i.e. the unobserved satellites are too big to not have visible stars [43]. Surprisingly, the $\mathrm{MeV}$ to $\mathrm{GeV}$ mass regime has so far remained largely unexplored.

Notable exceptions are variants of the SLIM (Scalar as Light as MeV) model, in which the SM is augmented at low energy by a scalar singlet and right-handed neutrinos [44] and in addition at higher energy e.g. by a complex doublet $[45,46]$. Original motivation for this model came from the fact that it could explain the $511 \mathrm{keV}$ emission line from the centre of our galaxy, observed by the INTEGRAL satellite [47]. If the scalar singlet is complex and almost as light as the lightest sterile neutrino of $\mathrm{MeV}$ mass, the latter can be the DM and not only lead approximately to the correct DM relic density and active neutrino masses, but also solve the missing satellite and too-big-to-fail problems [48]. In addition to structure formation constraints, this variant of the SLIM model has also passed collider constraints from LEP and the LHC, that we will review below, and is thus not only very well motivated, but also worthy of further investigation.

In this paper, we impose for the first time the precise Planck measurement of the DM relic density as well as the neutrino mass differences and mixing angles through the Casas-Ibarra parametrisation. In addition, we study in detail the most competitive lepton flavour violation constraints that had so far been overlooked in the literature, but turn out to be highly restrictive on the allowed parameter space due to the fact that the scalar doublet couples also to the charged leptons [49]. Since the DM is light and leptophilic, i.e. has no tree-level couplings to quarks, no visible signal from nuclear recoils is expected, although the latter might well be induced at one loop for GeV to TeV DM masses [50-52]. We therefore study instead the electron recoil signal, which has recently come into focus for sub-GeV DM [53-57] and should not only be sensitive to vector bosons (dark photons), but also other mediators, in particular the scalar doublet.

The goals of this paper are therefore threefold: first, after (re)defining the SLIM model with $\mathrm{MeV}$ neutrino DM in section 2 and reviewing in section 3 the theoretical and exper-

\footnotetext{
${ }^{1}$ See also references cited therein.
} 
imental constraints imposed previously, we calculate in section 4 the precise DM relic density from neutrino mass difference and mixing constraints without any analytic approximations. The result is not only the confirmation that neutrino DM is indeed viable in the $\mathrm{MeV}$ to $\mathrm{GeV}$ mass range, but also the prediction that its coupling to the scalar doublet can be large. Second, we compute in section 5 the expected lepton flavour violation signals in the most sensitive channels $\mu \rightarrow e \gamma, \mu \rightarrow 3 e$ and $\mu^{-} \mathrm{Ti} \rightarrow e^{-\mathrm{Ti}}$ and compare them with the sensitivities of current and future experiments. Third, we compute in section 6 the resulting electron recoil cross section and compare it with new, realistic estimates of the XENON sensitivity and other experiments under preparation. In section 7 we summarise our findings and discuss their generalisation to other models with MeV-scale DM.

\section{The SLIM model with MeV neutrino DM}

\subsection{Particle content}

In the SLIM model, the SM scalar (Higgs) doublet $\Phi$ with mass parameter $m_{1}$, self-coupling $\lambda_{1}$, squared vacuum expectation value $(\mathrm{VEV}) v^{2}=-2 m_{1}^{2} / \lambda_{1}=(246 \mathrm{GeV})^{2}$ and physical Higgs boson mass $m_{h}^{2}=\lambda_{1} v^{2}=(125 \mathrm{GeV})^{2}$ is augmented at low energy by a real or complex scalar singlet. While both options are in principle possible, we will discuss later the phenomenological advantages of our choice of a complex scalar

$$
\rho=\frac{1}{\sqrt{2}}\left(\rho_{R}+i \rho_{I}\right)
$$

and (at least) two generations of singlet right-handed neutrinos $N_{i}$ in order to effectively explain the observed DM and two non-zero neutrino masses at one loop [44]. The electroweak symmetry $\mathrm{SU}(2)_{L}$ is restored at higher energy by the addition of a complex scalar doublet $[45,46]$

$$
\eta=\left(\begin{array}{c}
\eta^{+} \\
\frac{1}{\sqrt{2}}\left(\eta_{R}+i \eta_{I}\right)
\end{array}\right) .
$$

All new particles are stabilised by a global U(1) symmetry that is softly broken to $Z_{2}$. At variance with the original scotogenic model, which was directly based on the $Z_{2}$ symmetry and contained only the complex scalar doublet and the right-handed neutrinos [36], the soft breaking of $\mathrm{U}(1)$ and the mixing of the scalar singlet and the neutral components of the scalar doublet, which both do not obtain a VEV, allow for MeV neutrino DM and two similarly light neutral scalars and thus for a solution to the missing satellite and too-big-to-fail problems [48]. In the classification of ref. [37], the scotogenic and SLIM models correspond to models T3-B and T1-1-A with hypercharge parameters $\alpha=-1$ and 0 , respectively.

\subsection{Lagrangian}

In the mass basis of the right-handed Majorana neutrinos $N_{i}(i=1,2)$ and denoting the left-handed SM lepton flavour doublets as $L_{j}(j=1,2,3)$, the Higgs potential and 
additional terms in the Lagrangian are given by $[45,46,48]$

$$
\begin{aligned}
\mathcal{L}= & -m_{1}^{2} \Phi^{\dagger} \Phi-m_{2}^{2} \eta^{\dagger} \eta-m_{3}^{2} \rho^{*} \rho-\frac{1}{2} m_{4}^{2}\left(\rho^{2}+\left(\rho^{*}\right)^{2}\right)-\mu\left(\eta^{\dagger} \Phi \rho+\text { h.c. }\right)-\frac{1}{2} m_{N_{i}} \overline{N_{i}^{c}} N_{i} \\
& -\frac{1}{2} \lambda_{1}\left(\Phi^{\dagger} \Phi\right)^{2}-\frac{1}{2} \lambda_{2}\left(\eta^{\dagger} \eta\right)^{2}-\frac{1}{2} \lambda_{3}\left(\rho^{*} \rho\right)^{2}-\lambda_{4}\left(\eta^{\dagger} \eta\right)\left(\Phi^{\dagger} \Phi\right)-\lambda_{5}\left(\eta^{\dagger} \Phi\right)\left(\Phi^{\dagger} \eta\right) \\
& -\lambda_{6}\left(\rho^{*} \rho\right)\left(\Phi^{\dagger} \Phi\right)-\lambda_{7}\left(\rho^{*} \rho\right)\left(\eta^{\dagger} \eta\right)-\left(\lambda_{8}\right)_{i j}\left(\overline{N_{i}^{c}} \eta^{\dagger} L_{j}+\text { h.c. }\right) .
\end{aligned}
$$

This DM model is leptophilic, as can be seen from the last term involving the couplings $\lambda_{8}$, and remains renormalisable despite the soft breaking of the global $\mathrm{U}(1)$ to $Z_{2}$, which implies that $m_{4}$ should be small.

\subsection{Scalar masses and mixings after electroweak symmetry breaking}

After electroweak symmetry breaking, the charged components of the scalar doublet obtain the mass

$$
m_{\eta^{ \pm}}^{2}=m_{2}^{2}+\frac{1}{2} \lambda_{4} v^{2}
$$

Due to the trilinear coupling $\mu$, the neutral components of the complex singlet and doublet mix, and one obtains the mass matrix

$$
M_{R, I}^{2}=\left(\begin{array}{cc}
m_{2}^{2}+\left(\lambda_{4}+\lambda_{5}\right) \frac{v^{2}}{2} & \mu \frac{v}{\sqrt{2}} \\
\mu \frac{v}{\sqrt{2}} & m_{3}^{2}+\lambda_{6} \frac{v^{2}}{2} \pm m_{4}^{2}
\end{array}\right)=:\left(\begin{array}{cc}
A & B \\
B & C_{R, I}
\end{array}\right)
$$

with a positive (negative) sign of the $m_{4}^{2}$ term for the real (imaginary) components. This leads to a very small mass splitting between the real and imaginary scalar mass eigenstates

$$
\left(\begin{array}{l}
\zeta_{1 R, I} \\
\zeta_{2 R, I}
\end{array}\right)=\left(\begin{array}{rr}
\cos \theta_{R, I} & -\sin \theta_{R, I} \\
\sin \theta_{R, I} & \cos \theta_{R, I}
\end{array}\right)\left(\begin{array}{l}
\eta_{R, I} \\
\rho_{R, I}
\end{array}\right)
$$

which are obtained by rotation about the angles $\theta_{R, I}$. The eigenvalues of the mass matrix are given by

$$
m_{R, I}^{2}=\frac{1}{2}\left(A+C_{R, I} \pm \sqrt{\left(A-C_{R, I}\right)^{2}+4 B^{2}}\right) .
$$

Setting $B^{2}$ close to $A C_{R, I}$ with only a small difference

$$
A C_{R, I}-B^{2}=: \epsilon\left(A+C_{R, I}\right)
$$

then allows to obtain two $\mathrm{MeV}$ neutral scalar mass eigenvalues $\left(m_{\zeta_{2 R, I}}\right)$, while the two others $\left(m_{\zeta_{1 R, I}}\right)$ will be of the same size as or larger than $m_{\eta^{ \pm}}$.

\section{Collider, cosmological and neutrino constraints}

Although the SLIM model spans a relatively large parameter space, the latter turns out to be restricted significantly both theoretically and experimentally. Starting with the Higgs potential, both of its parameters $\left(m_{1}\right.$ and $\left.\lambda_{1}\right)$ are now fixed by the known values of $v$ and $m_{h}$ (see section 2.1). Theoretically, unitarity requires certain combinations of the couplings 


\begin{tabular}{|c|c|c|l|}
\hline Parameter & Value & Range & Phenomenological impact \\
\hline$m_{1}^{2}$ & $-(89 \mathrm{GeV})^{2}$ & - & Fixed by $v$ and $m_{h}$ \\
$m_{2}$ & $83 \mathrm{GeV}$ & - & Fixed by $v, \lambda_{4}$ and $\lambda_{5}$ \\
$m_{3}$ & $264 \mathrm{GeV}$ & - & Fixed by $v$ and $\lambda_{6}$ \\
$m_{4}$ & - & $10 \mathrm{keV} \ldots 10 \mathrm{MeV}$ & Induces soft $\mathrm{U}(1)$ breaking \\
$\mu$ & - & $251 \mathrm{GeV} \ldots 252 \mathrm{GeV}$ & Fixed by $v$ and $\epsilon$ \\
$\epsilon$ & - & $\left(10^{-5} \ldots 6.1 \cdot 10^{1}\right) \mathrm{GeV}^{2}$ & Induces MeV masses \\
$m_{N_{1}}$ & - & $(0.1 \ldots 0.98) \cdot m_{\zeta_{2}}$ & $\mathcal{O}(\mathrm{MeV})$ DM candidate \\
$m_{N_{2}}$ & - & $10 \mathrm{GeV} \ldots 200 \mathrm{GeV}$ & $\mathcal{O}(\mathrm{GeV})$ sterile neutrino \\
\hline$\lambda_{1}$ & 0.26 & - & Fixed by $v$ and $m_{h}$ \\
$\lambda_{2}$ & 0.12 & - & Induces only scalar conversions \\
$\lambda_{3}$ & 0.13 & - & Induces only scalar conversions \\
$\lambda_{4}$ & 0.097 & - & Fixed by $v, m_{\eta^{ \pm}}=99 \mathrm{GeV}, R_{\gamma \gamma}$ \\
$\lambda_{5}$ & 0.13 & - & Fixed by $v, m_{\eta^{ \pm}}=99 \mathrm{GeV}, R_{\gamma \gamma}$ \\
$\lambda_{6}$ & 2.3 & - & Induces MeV masses \\
$\lambda_{7}$ & 0.17 & $10^{-6} \ldots 10^{2}$ & Induces only scalar conversions \\
$\lambda_{8}$ & - & Fixed by Casas-Ibarra parametr. \\
\hline
\end{tabular}

Table 1. Overview of model parameters with their values or scan ranges and phenomenological impact, respectively.

$\lambda_{k}(k=2, \ldots, 8)$ to lie below $8 \pi$, while vacuum stability and the need to avoid tachyonic particles require others to be positive or larger than certain squared mass differences. These theoretical constraints turn out to be almost automatically satisfied, once collider and cosmological constraints are imposed. Several mass parameters (in particular $m_{2,3}$ ) then also directly depend on some of the couplings $\left(\lambda_{4,5,6}\right)$. An overview of all model parameters, as defined by the Lagrangian in eq. (2.3), together with their values or scan ranges and phenomenological impact is given in table 1 . They are described in detail in the following.

\subsection{Collider constraints}

The SM scalar Higgs doublet $\Phi$ couples to the new complex scalar doublet $\eta$ and singlet $\rho$ through the matrix

$$
\left(\begin{array}{c}
\eta \\
\rho
\end{array}\right)^{\dagger}\left(\begin{array}{cc}
\left(\lambda_{4}+\lambda_{5}\right) v & \frac{\mu}{\sqrt{2}} \\
\frac{\mu}{\sqrt{2}} & \lambda_{6} v
\end{array}\right) \Phi\left(\begin{array}{l}
\eta \\
\rho
\end{array}\right) .
$$

Inspection of eq. (2.5) shows that, for negligibly small $m_{4}$, the identifications [48]

$$
m_{2}^{2}=\left(\lambda_{4}+\lambda_{5}\right) \frac{v^{2}}{2} \quad \text { and } \quad m_{3}^{2}=\lambda_{6} \frac{v^{2}}{2}
$$

make the Higgs coupling matrix proportional to the scalar mass matrix. While the invisible decays $h \rightarrow \zeta_{1 R, I} \zeta_{1 R, I}$ can be forbidden by choosing $m_{\zeta_{1 R, I}}>m_{h} / 2$, the decays $h \rightarrow$ 
$\zeta_{2 R, I} \zeta_{2 R, I} \rightarrow N_{1} N_{1} \nu \nu$ will generally be kinematically open due to the small values of $m_{\zeta_{2 R, I}}$, but then also have negligible branching ratios. As a consequence, $m_{2,3}$ are fixed by $\lambda_{4,5,6}$. Experimentally, the current ATLAS and CMS limits on the invisible Higgs branching ratio lie at $26 \%$ and $19 \%$, respectively $[58,59]$.

A linear combination of the parameters $\lambda_{4,5}$ can in turn be restricted by observing that the charged scalar mass $m_{\eta^{ \pm}}$in eq. (2.4) must lie above the rather model-independent LEP limit of $98.5 \mathrm{GeV}$ for charged scalars [60]. The charged scalar also contributes to the Higgs branching ratio into two photons, for which CMS now measures a ratio to the SM value of $R_{\gamma \gamma}=1.20_{-0.14}^{+0.18}$ [61]. Choosing $\lambda_{4,5} \simeq 0.1\left(\lambda_{4}=0.097, \lambda_{5}=0.13\right)$ satisfies both constraints, as we have verified and can also be seen from figure 3 in ref. [48]. Larger values of $\lambda_{4}$ would lead to larger $m_{\eta^{ \pm}}$and thus smaller electron recoil cross sections and lepton flavour violation, while $\lambda_{5}$ has similar, but somewhat less phenomenological influence.

\subsection{Cosmological constraints}

Although studies of the SLIM model initially focused on the possibility of scalar MeV DM [44-46], also to explain the $511 \mathrm{keV}$ line from the galactic centre [47], it was later noticed that the problems of missing satellite galaxies, the cusp core of inner DM density profiles and the fact that the unobserved satellites are too big to not have visible stars [43] can rather be solved with $\mathrm{MeV}$ neutrino $\mathrm{DM}$, provided that it scatters off the active neutrinos in the early Universe by exchanging only slightly heavier scalars [48].

In the scalar sector, these cosmological constraints translate into singlet and doublet mass parameters that should be close to each other, so that the cancellation described in section 2.3 can occur. While $\lambda_{2,3,7}$ only control the scalar potentials and the conversion of scalars into each other with little influence on the phenomenology (we take e.g. $\lambda_{2}=0.12$, $\lambda_{3}=0.13$, and $\lambda_{7}=0.17$ ), the singlet mass parameter $m_{3}$ should not be much larger than $v$ (i.e. $\lambda_{6} \leq 3$ ), as was also observed in figure 2 of ref. [48]. Instead of varying $\lambda_{6}$ and fixing $\epsilon$ to $10^{-4} \mathrm{GeV}^{2}$, we rather fix $\lambda_{6}=2.3$ and vary the mass splitting parameter in eq. (2.8)

$$
\epsilon=\left(10^{-5} \ldots 6.1 \cdot 10^{1}\right) \mathrm{GeV}^{2} .
$$

This has the advantage of more directly controlling the off-diagonal mass parameter $\mu$ and thus the MeV mass of the two light scalars $\zeta_{2 R, I}$.

It has been shown in refs. $[62,63]$ that weak-strength DM interactions can erase the DM primordial fluctuations when the DM is relatively light (of $\mathcal{O}(\mathrm{MeV})$ ) and coupled to neutrinos or photons. The elastic scattering cross section must then, however, be temperatureindependent, which requires, in addition to $\mathrm{MeV}$ DM mass and weak-strength DM couplings, a small mass splitting of the MeV neutrino DM and the lightest scalar. This is ensured in all our scenarios through the imposed scan ranges, in particular of $m_{4}, \epsilon$ and $\lambda_{6}$.

For the lighter Majorana neutrino $N_{1}$ to represent DM and solve the cosmological problems, it must therefore be (only somewhat) lighter than the lightest scalar. We therefore vary $m_{N_{1}} / m_{\zeta_{2 R, I}}$ in the range $0.1 \ldots 0.98$. In contrast, when the second Majorana neutrino $N_{2}$ is heavy $(10 \mathrm{GeV} \ldots 200 \mathrm{GeV}$, the exact value being constrained by the active neutrino mass differences and mixings), it decays promptly through $N_{2} \rightarrow \eta \nu \rightarrow N_{1} \nu \nu$ and 


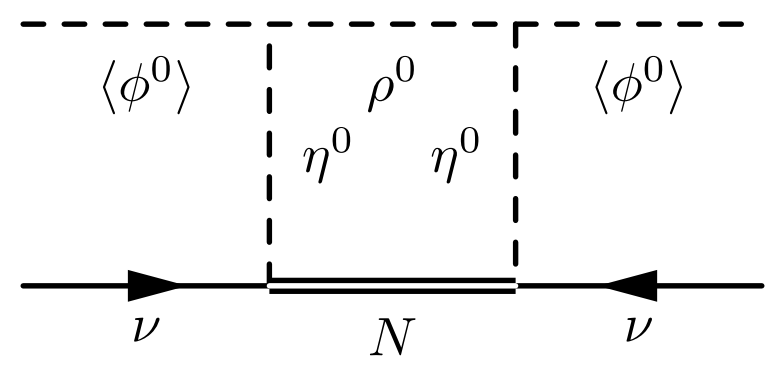

Figure 1. One-loop diagram leading to the active neutrino mass matrix in the SLIM model.

contributes neither significantly to the relic density nor to any other signal considered here. A third Majorana neutrino could of course be added, but would not significantly change the phenomenology.

\subsection{Neutrino constraints}

In radiative seesaw models, the neutrinos obtain their masses only at the loop level, and in particular in the SLIM model through the one-loop diagram shown in figure 1.

Taking the mixing in the neutral scalar sector as described in section 2.3 into account, the neutrino mass matrix is given by [48]

$$
\begin{aligned}
\left(M_{\nu}\right)_{i j}=\sum_{k} \frac{\left(\lambda_{8}\right)_{i k}\left(\lambda_{8}\right)_{j k}}{16 \pi^{2}} m_{N_{k}}[ & \frac{\cos ^{2} \theta_{R} m_{1 R}^{2}}{m_{1 R}^{2}-m_{N_{k}}^{2}} \ln \frac{m_{1 R}^{2}}{m_{N_{k}}^{2}}+\frac{\sin ^{2} \theta_{R} m_{2 R}^{2}}{m_{2 R}^{2}-m_{N_{k}}^{2}} \ln \frac{m_{2 R}^{2}}{m_{N_{k}}^{2}} \\
& \left.-\frac{\cos ^{2} \theta_{I} m_{1 I}^{2}}{m_{1 I}^{2}-m_{N_{k}}^{2}} \ln \frac{m_{1 I}^{2}}{m_{N_{k}}^{2}}-\frac{\sin ^{2} \theta_{I} m_{2 I}^{2}}{m_{2 I}^{2}-m_{N_{k}}^{2}} \ln \frac{m_{2 I}^{2}}{m_{N_{k}}^{2}}\right] .
\end{aligned}
$$

As in the scotogenic model, the neutrino masses arise from the small mass differences between the real and imaginary scalars. In the SLIM model, this mass splitting is realised by the non-zero value of $m_{4}$ (cf. section 2.3), required to be small from the soft breaking of $\mathrm{U}(1)$ to $Z_{2}$. We do not fix $m_{4}$ here to the relatively large value of $3 \mathrm{MeV}$ as in ref. [48], but allow it to vary in the range $10 \mathrm{keV} \ldots 10 \mathrm{MeV}$, as a smaller mass splitting of $\zeta_{2 R, I}$ reduces the mass-dependent terms in the neutrino mass matrix and thus indirectly allows for larger lepton couplings $\lambda_{8}$.

We take the neutrino mass and mixing constraints into account assuming for simplicity a massless lightest neutrino, which implies a normal hierarchy and absolute values for the other two neutrino masses in the mass matrix

$$
D_{\nu}=U_{\nu}^{\dagger} M_{\nu} U_{\nu}=\operatorname{diag}\left(0, m_{\nu 2}, m_{\nu 3}\right),
$$

diagonalised by the PMNS matrix $U_{\nu}$.

To impose the experimental constraints on the neutrino mass and mixing, we make use of the Casas-Ibarra parametrisation [64]. It takes as an input the experimental neutrino data and the dark particle masses and mixings and returns the coupling $\lambda_{8}$. Specifically, we rewrite eq. (3.4) as

$$
M_{\nu}=\lambda_{8}^{T} M \lambda_{8}
$$


where $M$ is a $3 \times 3$ diagonal matrix whose elements are differences of mass functions, summed over $k$. Together with eq. (3.5) we then obtain

$$
D_{\nu}=U_{\nu}^{\dagger} \lambda_{8}^{T} M \lambda_{8} U_{\nu}
$$

or

$$
\operatorname{diag}(0,1,1)=D_{\nu}^{-1 / 2} U_{\nu}^{\dagger} \lambda_{8}^{T} M \lambda_{8} U_{\nu} D_{\nu}^{-1 / 2} \equiv R^{\dagger} R,
$$

where the rotation angle $\theta$ in

$$
R=\left(\begin{array}{ccc}
0 & \cos \theta & \sin \theta \\
0 & -\sin \theta & \cos \theta
\end{array}\right)
$$

is arbitrary, i.e. it can vary in the range $0 \ldots 2 \pi$. Identifying

$$
R=M^{1 / 2} \lambda_{8} U_{\nu} D_{\nu}^{-1 / 2}
$$

then leads to the desired couplings

$$
\lambda_{8}=M^{-1 / 2} R D_{\nu}^{1 / 2} U_{\nu}^{\dagger},
$$

that are consistent with the neutrino data and depend not only on $\theta$, but also indirectly on the scalar and right-handed neutrino masses as well as the scalar couplings in the potential. The coupling $\lambda_{8}$ itself determines many properties of the model. Apart from being responsible for the neutrino masses, it also directly influences the relic density, the electron recoil cross section, as well as the amplitude of lepton flavour violating processes such as $\mu \rightarrow e \gamma$.

\section{Right-handed neutrino DM relic density, mass and lepton couplings}

We now come to the first new result of our study, i.e. a numerical calculation of the precise right-handed neutrino DM relic density without any analytic approximations. To this end, we reproduce the Lagrangian of the SLIM model [45, 46, 48] presented in eq. (2.3) from the particle content defined in section 2.1 using our program MINIMAL-LAGRANGIANS [65]. The corresponding Feynman rules are then derived with SARAH 4.14.2 [66] and passed on to SPheno 4.0.3 [67] to calculate the physical mass spectrum for each point in the parameter space that we have defined in section 3.

At this point, we do not only impose the collider, cosmological and neutrino constraints in the ways described there, but also use SPHENo 4.0.3 to check (again) other collider and low-energy constraints such as the $Z$ and Higgs boson branching ratios, the $\rho$ parameter, and the braching ratio of the flavour-changing neutral current process $b \rightarrow s \gamma$. The right-handed neutrino DM relic density for each surviving model is then calculated with MICROMEGAS 4.3.5 [68].

Figure 2 shows the DM relic density $\Omega h^{2}$ of all viable SLIM models with right-handed neutrino DM versus the absolute value of its coupling to electrons and electron neutrinos $\lambda_{8}^{e 1}$, coloured according to the value of the mass $m_{N_{1}}$ of the DM candidate. One can clearly see that the relic density drops quickly from $10^{0}$ to $10^{-4}$ when the lepton coupling 


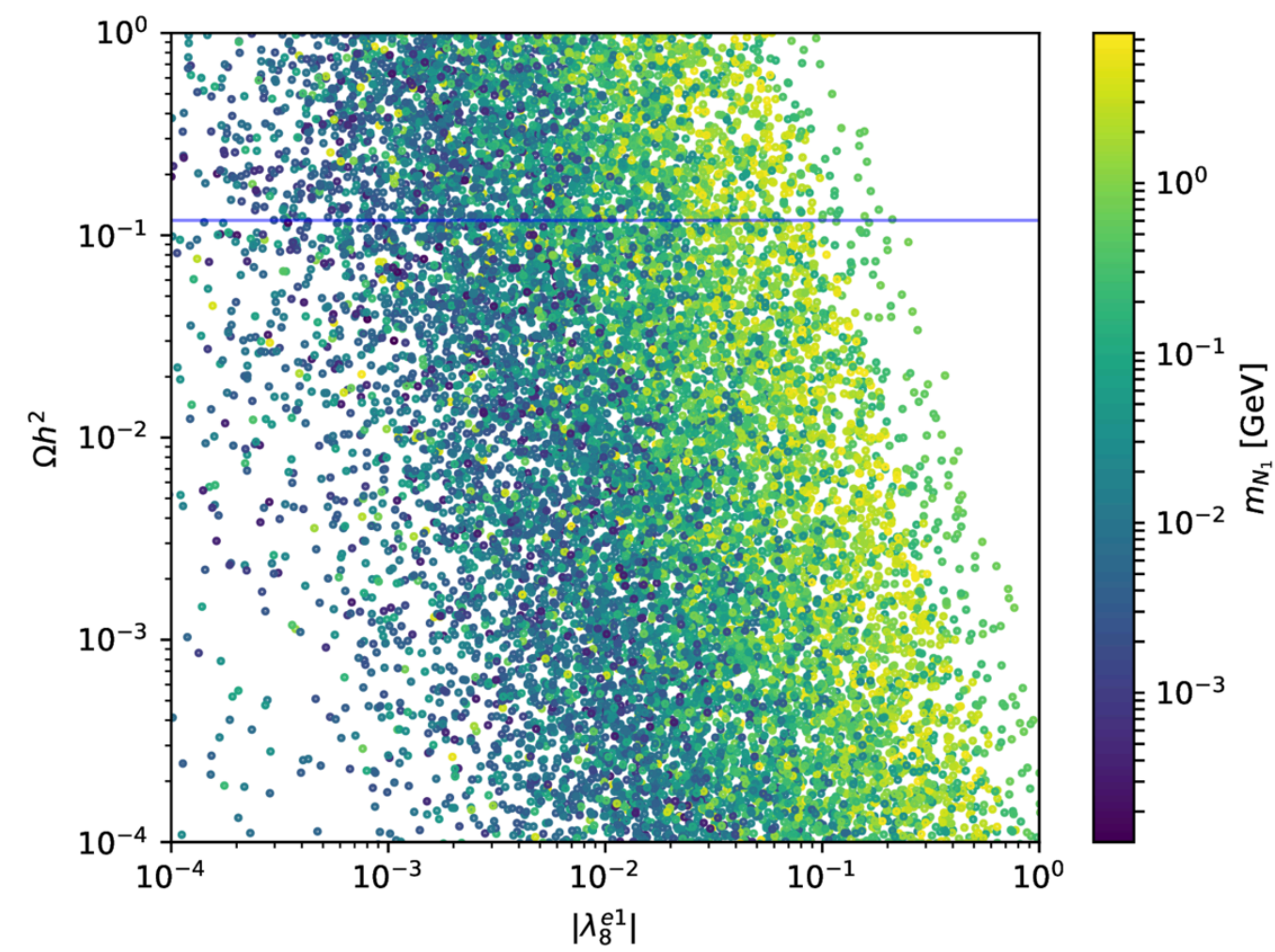

Figure 2. The relic density $\Omega h^{2}$ obtained in the SLIM model with right-handed neutrino DM versus the absolute value of its coupling to electrons and electron neutrinos $\lambda_{8}^{e 1}$. The relic density measured by Planck [2] is shown as a blue horizontal band, and the lightest Majorana neutrino mass $m_{N_{1}}$ is given on a colour scale.

rises by about two orders of magnitude, e.g. from a few times $10^{-4}$ to $10^{-2}$ or $10^{-2}$ to a few times $10^{-1}$. However, the correct relic density as observed by Planck (blue horizontal band) [2] can be obtained for all couplings in these ranges, provided that the DM mass rises simultaneously from the $\mathrm{MeV}$ (dark blue) to the GeV scale (light green/yellow). We have therefore confirmed that while $\mathrm{MeV}$ dark matter is indeed consistent with collider, structure formation and neutrino constraints, the correct relic density imposes further restrictions and in fact predicts sizeable couplings of almost up to $\mathcal{O}(1)$ to the SM leptons. In the following, we therefore impose also the Planck value for the relic density.

In figure 3 we show all models, which satisfy not only collider, cosmological and neutrino mass constraints, but also lead to the correct relic density. It therefore represents a projection of figure 2 on the blue line and is displayed in the plane of the absolute value of the DM-electron coupling $\left|\lambda_{8}^{e 1}\right|$ versus DM mass $m_{N_{1}}$. As one can observe, heavier DM implies enhanced DM annihilation processes through larger couplings to the SM leptons in order to reduce the DM abundance and still obtain the correct relic density. 


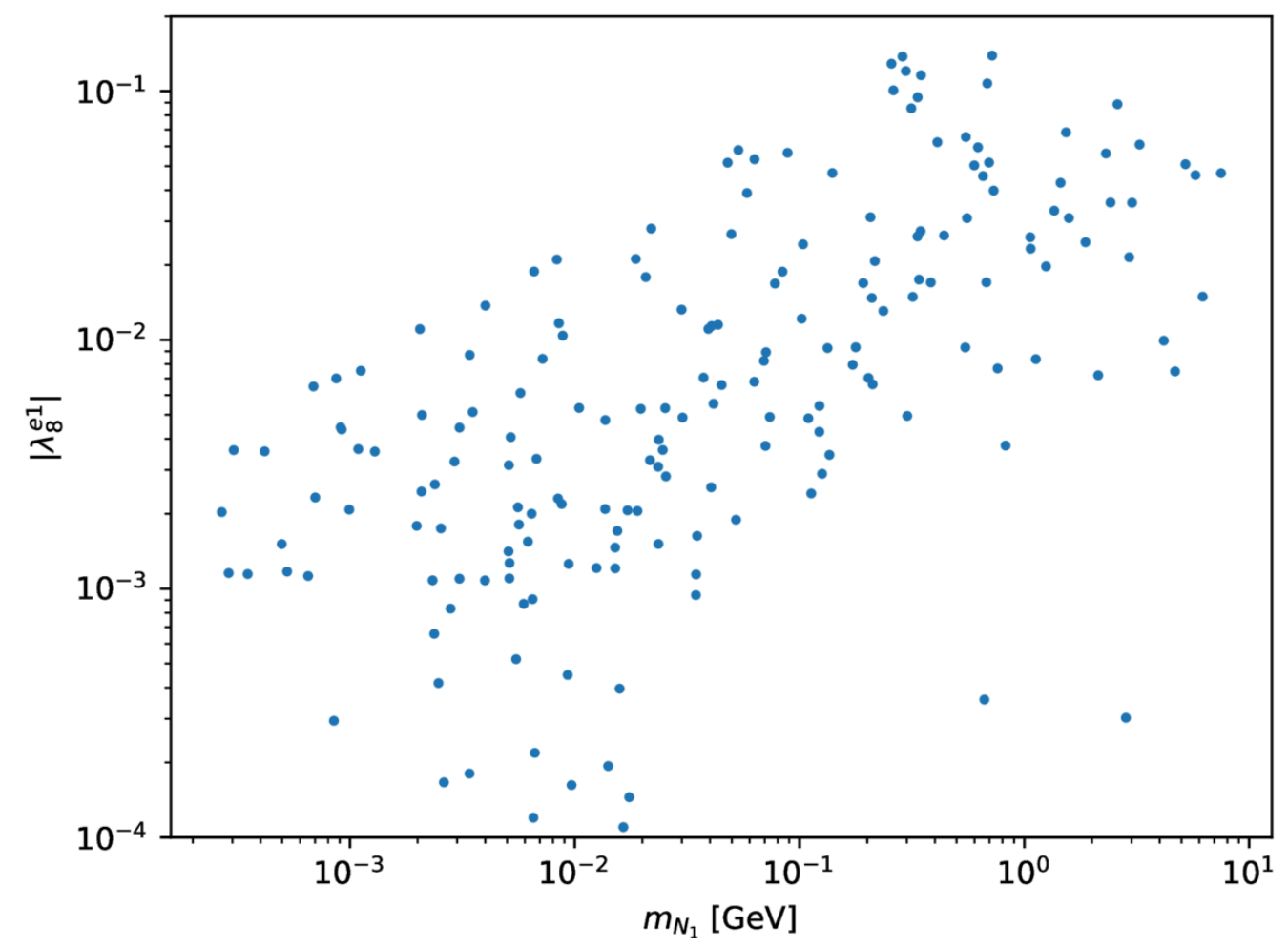

Figure 3. DM-electron coupling $\lambda_{8}^{e 1}$ versus DM mass $m_{N_{1}}$ for models which satisfy not only collider, cosmological and neutrino mass constraints, but also lead to the correct relic density.

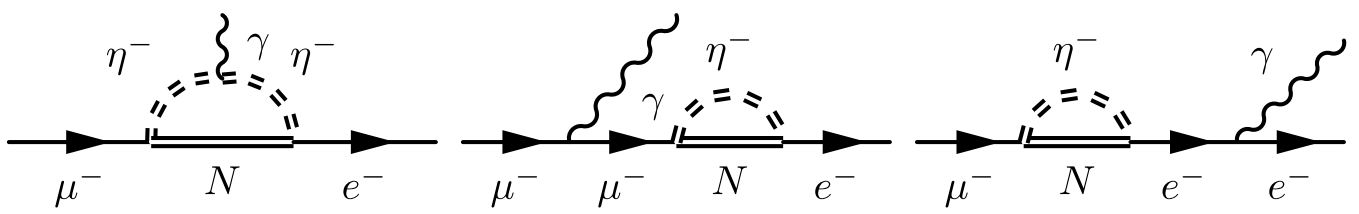

Figure 4. Feynman diagrams contributing to the lepton flavour violating process $\mu \rightarrow e \gamma$ at one loop through the exchange of $Z_{2}$-odd right-handed neutrinos $(N)$ and charged scalars $\left(\eta^{-}\right)$.

\section{Lepton flavour violation and experimental constraints}

Since the right-handed neutrinos $N_{i}$ in our model couple through the complex scalar doublet $\eta$ not only to SM neutrinos, but also to the charged leptons, processes that violate lepton flavour naturally occur at the one-loop level. The most sensitive process with the strictest experimental bounds is usually the flavour-changing neutral current process $\mu \rightarrow e \gamma$. Here, it occurs through the Feynman diagrams shown in figure 4 and depends, apart from the exchanged particle masses, not only on the couplings $\lambda_{8}^{e i}$, but also on $\lambda_{8}^{\mu i}$, which are both constrained by the neutrino masses and mixings through the Casas-Ibarra parametrisation. 


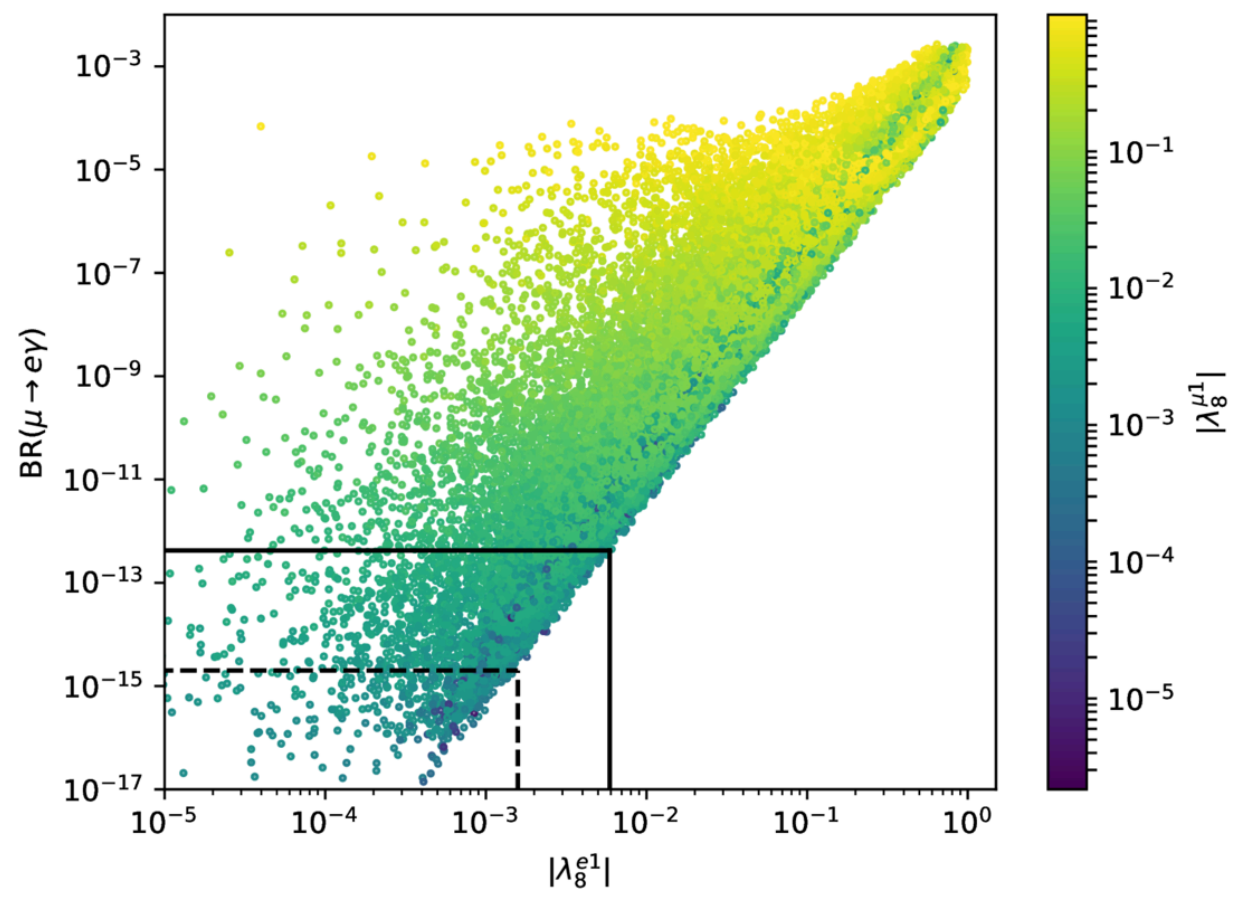

Figure 5. Branching ratio of the lepton flavour violating process $\mu \rightarrow e \gamma$ versus the DM-electron coupling $\lambda_{8}^{e 1}$. The DM-muon coupling $\lambda_{8}^{\mu 1}$ is shown on a colour scale. Also shown are the current (solid black line) [69] and expected future (dashed black line) [70] limits on the branching ratio by the MEG experiment.

We compute the branching ratio of the process $\mu \rightarrow e \gamma$ with SPHENo 4.0.3 [67] and plot it in figure 5 as a function of the dominating couplings $\lambda_{8}^{e 1}$ on the $x$-axis and $\lambda_{8}^{\mu 1}$ on a colour scale. Interestingly, the non-zero neutrino masses impose a lower bound on this branching ratio of about $10^{-17}$. Experimentally, the MEG experiment currently excludes branching ratios above $4.2 \cdot 10^{-13}$ [69]. This already excludes a substantial part of the parameter space and in particular couplings $\lambda_{8}^{e 1}$ above $6 \cdot 10^{-3}$ and $\lambda_{8}^{\mu 1}$ above about $10^{-2}$. In the SLIM model with scalar DM, similar limits of a few times $10^{-3}$ and a few times $10^{-2}$, respectively, have been expected in an approximate calculation [45, 46]. After the planned upgrade of the MEG experiment, it is expected to reach a sensitivity of $2 \cdot 10^{-15}$ [70], which would lower the limits on the couplings by about a factor of four, leaving open only a small part of the parameter space.

The current limit on the branching ratio $\mu \rightarrow 3 e$ obtained by the SINDRUM experiment lies at $10^{-12}$ [71]. It is thus only slightly weaker than the MEG limit on $\mu \rightarrow e \gamma$. In addition, SINDRUM II obtained a limit of $4.3 \cdot 10^{-12}$ on the muon-to-electron conversion rate in Titanium [72]. Significant progress is expected for both processes with possible future limits of $10^{-16}$ [73] and even $10^{-18}$ [74], respectively. In figure 6 we therefore show predictions for these processes in our viable models, satisfying all collider, cosmological and 


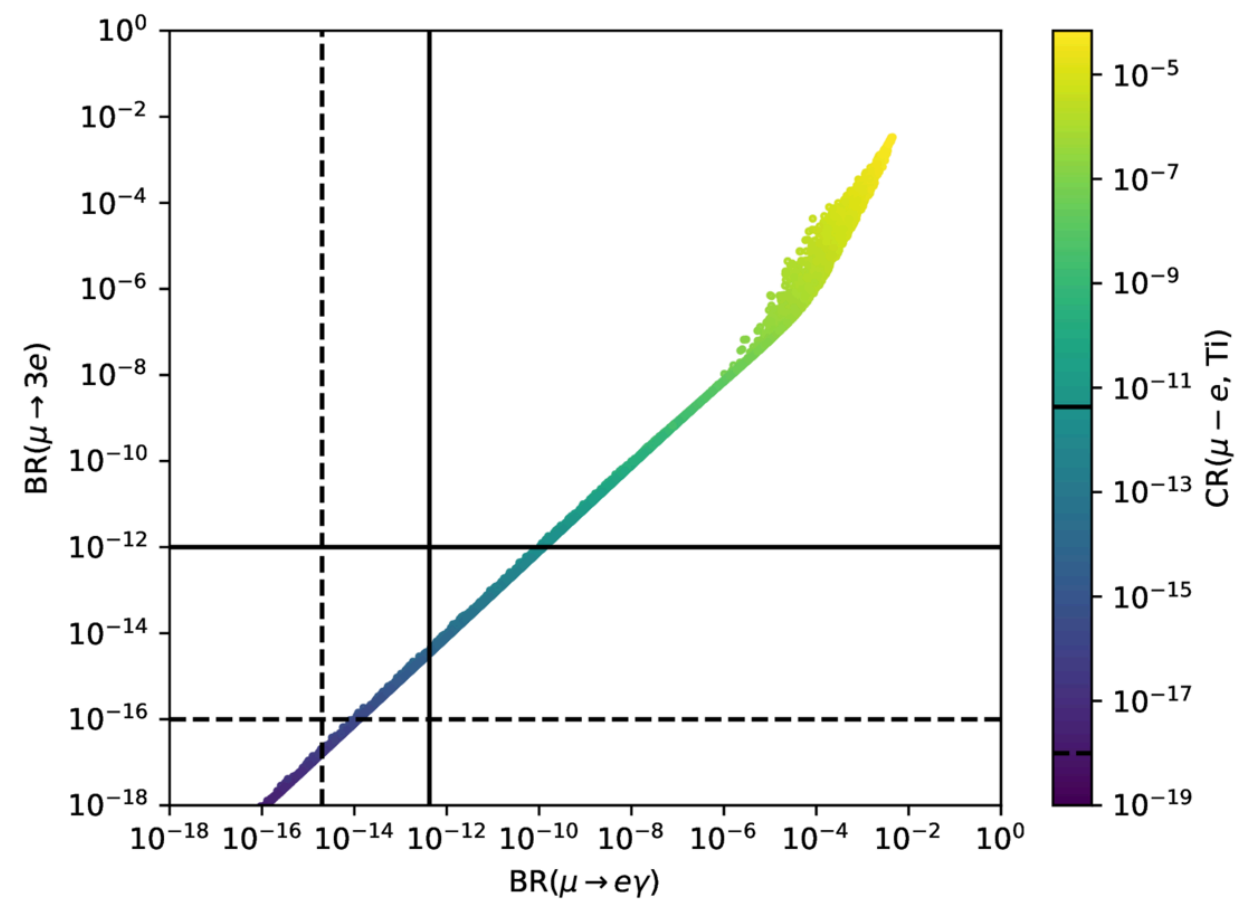

Figure 6. Branching ratio of the lepton flavour violating process $\mu \rightarrow 3 e$ versus the one of the (usually most constraining) process $\mu \rightarrow e \gamma$. The rate of muon conversion in the field of titanium atoms $\mu^{-} \mathrm{Ti} \rightarrow e^{-} \mathrm{Ti}$ is shown on a colour scale. Also shown are the current (solid black lines) $[69,71,72]$ and expected future (dashed black lines) $[70,73,74]$ experimental limits on the branching ratios and conversion rate.

neutrino constraints, together with those for $\mu \rightarrow e \gamma$ and compare them to the current (solid lines) and future (dashed lines) experimental limits. We observe a strong correlation of all three processes, except for large, already excluded branching ratios and conversion rates. While the current limit on $\mu \rightarrow e \gamma$ sets the strongest bound, the other two experiments will indeed reach a similar sensitivity in the future. Note that the scans in figures 5 and 6 do not include the constraint on the correct relic density, i.e. also points which under- (or over-) saturate the relic density are included.

\section{Electron recoil cross section and experimental sensitivity}

As described in the introduction, the DM in the SLIM model is leptophilic, so that one does not expect any nuclear recoil signal from experiments like XENON1T, which has set the current best limit on the spin-independent direct detection cross section for DM masses between $6 \mathrm{GeV}$ and $1 \mathrm{TeV}$ [28], XENON100, which had extended the sensitivity down to $3.7 \mathrm{GeV}$ using the ionisation (S2) signal only [75], or CRESST-III, whose cryogenic $\mathrm{CaWO}_{4}$ crystals are even sensitive down to DM masses of $160 \mathrm{MeV}[76]$. 
Given that the $\mathrm{DM}$ is of $\mathrm{MeV}$ to $\mathrm{GeV}$ mass and its couplings to the SM leptons are large, one would, however, expect an observable electron recoil signal, which has recently come into focus for sub-GeV DM and should not only be sensitive to vector bosons (dark photons), but also other mediators [53-57]. If we generalise the notation for the DM particle to the conventional $\chi$, the ionisation rate, differential in the electron recoil (er) energy $E_{\text {er }}$,

$$
\frac{d R_{\mathrm{ion}}}{d \ln E_{\mathrm{er}}}=N_{\mathrm{T}} \frac{\rho_{\chi}}{m_{\chi}} \sum_{n \ell} \frac{d\left\langle\sigma_{\mathrm{in}}^{n \ell} v\right\rangle}{d \ln E_{\mathrm{er}}}
$$

is proportional to the number of target nuclei per unit mass $N_{T}$, the local DM density $\rho_{\chi}=0.4 \mathrm{GeV} / \mathrm{cm}^{3}[77]$, the differential thermally averaged cross section

$$
\frac{d\left\langle\sigma_{\mathrm{ion}}^{n \ell} v\right\rangle}{d \ln E_{\mathrm{er}}}=\frac{\bar{\sigma}_{\chi \mathrm{e}}}{8 \mu_{\chi \mathrm{e}}^{2}} \int\left|f_{\mathrm{ion}}^{n \ell}\left(k^{\prime}, q\right)\right|^{2} F\left(k^{\prime}, Z_{\mathrm{eff}}\right)\left|F_{\mathrm{DM}}(q)\right|^{2} \eta\left(v_{\min }, t\right) q d q,
$$

summed over all possible electron states $(n, \ell)$, and inversely proportional to the DM mass $m_{\chi}$. In eq. (6.2), $\mu_{\chi \mathrm{e}}$ is the DM-electron reduced mass, and $k^{\prime}=\sqrt{2 m_{e} E_{\text {er }}}$ and $q$ are the outgoing electron momentum and momentum transfer, respectively. Assuming plane waves for the scattered electrons and a spherically symmetric atom with full shells, the form factor for ionisation reduces to

$$
\left|f_{\text {ion }}^{n \ell}\left(k^{\prime}, q\right)\right|^{2}=\frac{(2 \ell+1) k^{\prime 2}}{4 \pi^{3} q} \int\left|\chi_{n \ell}(k)\right|^{2} k d k
$$

where $\chi_{n \ell}(k)$ is the radial part of the momentum space wave function of the bound electron [50-52]. As in the case of nuclear beta decay, the wave function of the escaping electron is distorted by the presence of the nearby atom, requiring that the rate be corrected by the Fermi function

$$
F\left(k^{\prime}, Z_{\text {eff }}\right)=\frac{2 \pi \nu}{1-e^{-2 \pi \nu}} \quad \text { with } \quad \nu=Z_{\text {eff }} \frac{\alpha m_{e}}{k^{\prime}} .
$$

$Z_{\text {eff }}$ is the effective charge felt by the electron, expected to be somewhat larger than unity. However, for outer shell electrons $Z_{\text {eff }}=1$ is a good approximation [53-57]. Treating the electrons in the target as single-particle states of an isolated atom, we can use the tabulated numerical Roothaan-Hartree-Fock (RHF) wave functions [78]. Astrophysics enters through the mean inverse DM velocity [57]

$$
\eta\left(v_{\min }, t\right)=\int_{v_{\min }}^{\infty} \frac{d^{3} v}{v} f(\mathbf{v}, t)
$$

which depends on the Earth-frame velocity distribution of DM $f(\mathbf{v}, t)$, that acquires a time dependence as the Earth orbits the Sun. In the Galactic frame, and asymptotically far away from the Sun's gravitational potential, we take the velocity distribution $f_{\infty}(\mathbf{v})$ to be that of the Standard Halo Model

$$
f_{\infty}(\mathbf{v})=1 / N_{\mathrm{esc}}\left(\pi v_{0}^{2}\right)^{-3 / 2} e^{-\mathbf{v}^{2} / v_{0}^{2}}, \quad \text { if } \quad|\mathbf{v}| \leq v_{\mathrm{esc}},
$$



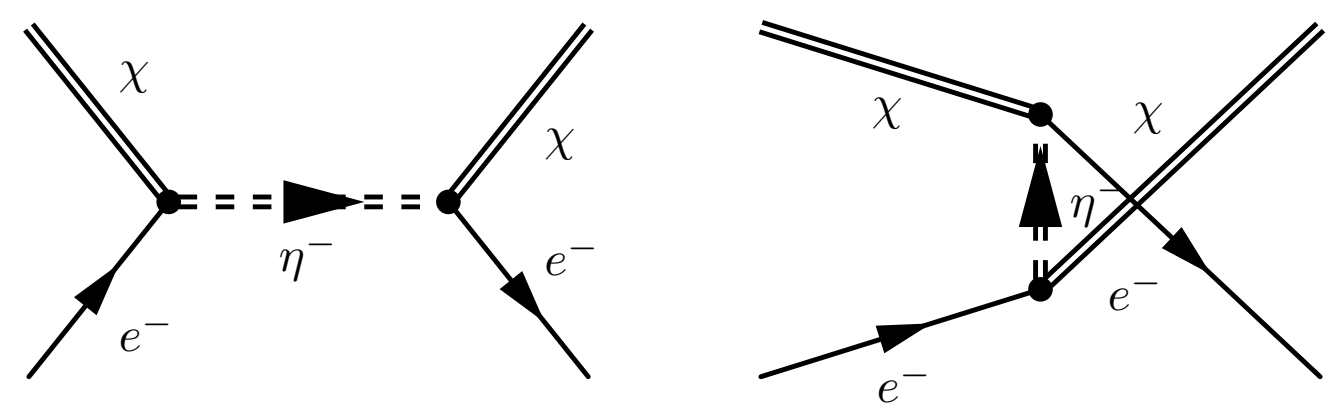

Figure 7. Feynman diagrams contributing to the scattering of right-handed neutrino DM $\left(\chi=N_{1}\right)$ off electrons $\left(e^{-}\right)$through $s$ - and $u$-channel exchanges of the charged $Z_{2}$-odd scalars $\left(\eta^{-}\right)$.

where $N_{\text {esc }}$ is a normalisation factor. We adopt a local circular velocity of $v_{0}=220 \mathrm{~km} / \mathrm{s}$ and an escape velocity of $v_{\text {esc }}=544 \mathrm{~km} / \mathrm{s}$ [79]. The minimal DM velocity

$$
v_{\min }=\frac{E_{\mathrm{B}}^{n \ell}+E_{\mathrm{er}}}{q}+\frac{q}{2 m_{\chi}}
$$

depends on the required sum of binding energy $E_{\mathrm{B}}^{n \ell}$ and recoil energy $E_{\mathrm{er}}$.

\subsection{Theoretical expectations}

In eq. (6.2), we are of course primarily interested in the DM scattering cross section off electrons. It is usually factorised into the non-relativistic reference cross section

$$
\bar{\sigma}_{\chi \mathrm{e}}=\left.\frac{\mu_{\chi \mathrm{e}}^{2}}{16 \pi m_{\chi}^{2} m_{\mathrm{e}}^{2}} \overline{\left|\mathcal{M}_{\mathrm{e} \chi}(q)\right|^{2}}\right|_{q^{2}=\alpha^{2} m_{\mathrm{e}}^{2}}
$$

at fixed momentum transfer $q=\alpha m_{\mathrm{e}}$, where $\alpha$ is the electromagnetic fine structure constant and $m_{e}$ is the electron mass, and the form factor

$$
\left|F_{\mathrm{DM}}(q)\right|^{2}=\overline{\left|\mathcal{M}_{\mathrm{e} \chi}(q)\right|^{2}} / \overline{\left|\mathcal{M}_{\mathrm{e} \chi}\left(\alpha m_{\mathrm{e}}\right)\right|^{2}},
$$

that captures the $q$-dependence of the matrix element. For light mediators, $F_{\mathrm{DM}}(q)=$ $\alpha^{2} m_{e}^{2} / q^{2}$, whereas for heavy mediators $F_{\mathrm{DM}}(q)=1$. In our model, the DM particles $\chi$ are the right-handed neutrinos $N_{1}$. They scatter off the electrons through the exchange of the $Z_{2}$-odd scalars $\eta^{-}$, coupling with strength $\lambda_{8}^{e 1}$, in the $s$ - and $u$-channel, as shown in the Feynman diagrams in figure 7. Since the charged scalar $\eta^{-}$is heavy (cf. section 3.2), the propagator can be integrated out with the result that the form factor $F_{\mathrm{DM}}(q)=1$ and the reference cross section is

$$
\bar{\sigma}_{\chi \mathrm{e}}=\frac{\mu_{\chi e}^{2}\left(\lambda_{8}^{e 1}\right)^{4}}{\pi m_{\eta^{ \pm}}^{4}} .
$$

For the models satisfying all the constraints described in section 3 and reproducing the observed DM relic density as described in section 4, the numerical values of the scattering cross sections of right-handed neutrino DM off electrons are shown in figure 8 as a function of the DM mass. In addition, the size of the DM-electron coupling $\lambda_{8}^{e 1}$ is shown on a 


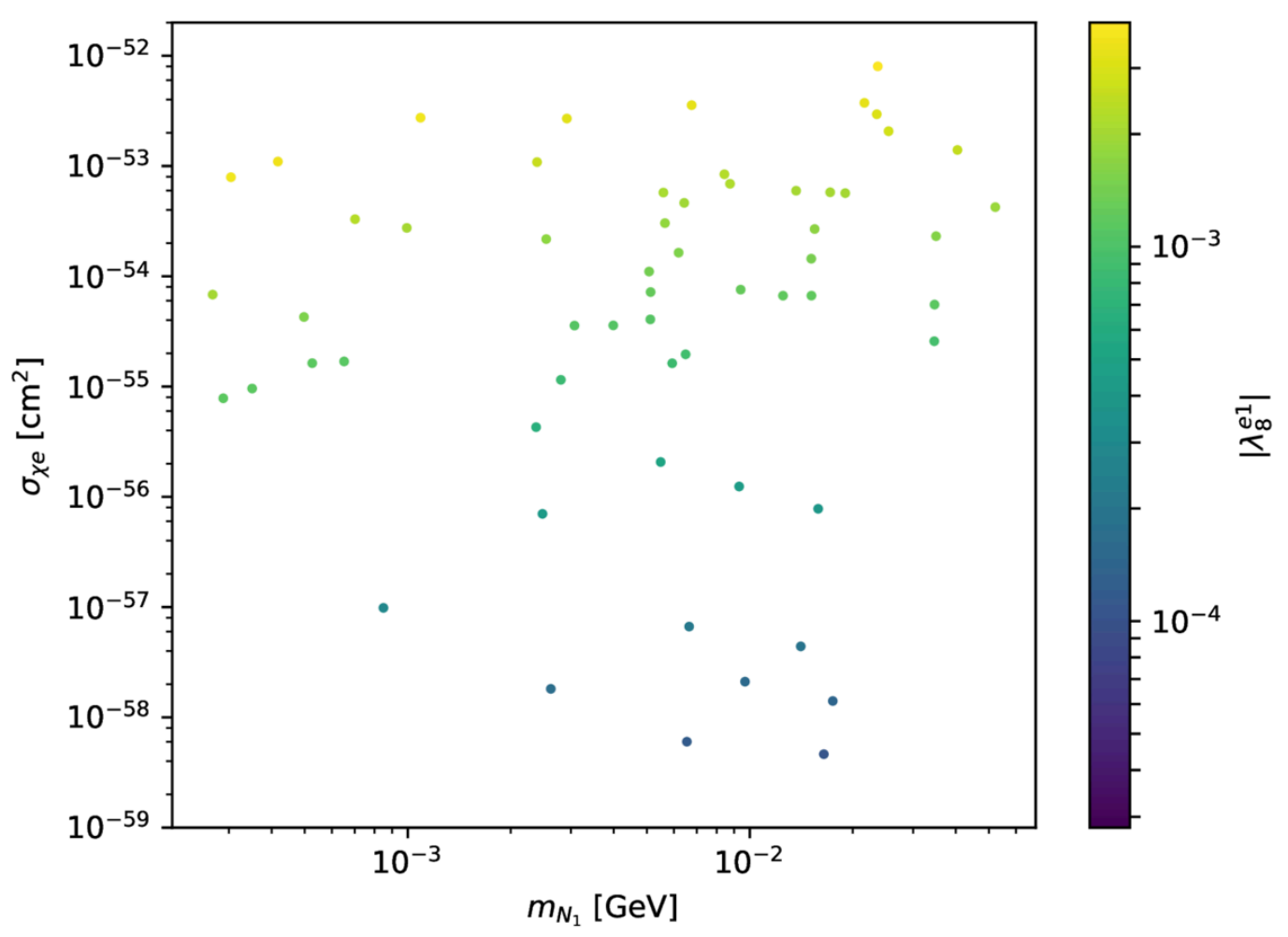

Figure 8. Scattering cross section of right-handed neutrino DM off electrons versus the DM mass. The DM-electron coupling $\lambda_{8}^{e 1}$ is shown on a colour scale.

colour scale. The cross sections do not exceed a few times $10^{-46} \mathrm{~cm}^{2}$ even for the largest couplings $\lambda_{8}^{e 1} \geq 0.1$ and reach only $10^{-52} \mathrm{~cm}^{2}$, when in addition the lepton flavour violation constraints of section 5 are imposed. The reason is the strong suppression by the large mass $m_{\eta^{ \pm}}$of the charged scalar mediator, which is constrained by the LEP limit to be heavier than $98.5 \mathrm{GeV}$ (cf. section 3.1) and which enters with the fourth power in eq. (6.10). Note that most of our viable models have DM masses above a few $\mathrm{MeV}$, which is in excellent agreement with bounds on big-bang nucleosynthesis (BBN) [80].

\subsection{Experimental sensitivity}

For DM with sub-GeV mass and no nuclear interactions, direct detection experiments must rely on the recoil of (one or several) electrons leading to ionisation. The cross section sensitivity of an experiment with a liquid xenon (LXe) target and an exposure of $1 \mathrm{~kg}$-year, assuming a constant form factor, no detector threshold and only the irreducible neutrino background, was initially estimated to be a few times $10^{-41} \mathrm{~cm}^{2}$ for masses of a few tens of $\mathrm{MeV}$ [53-56]. In figure 9 we plot this sensitivity (red dotted line) for an exposure of 60 kg-year by simply scaling it down by a factor of 60 , neglecting the influence of the tiny but non-zero irreducible background of neutrino scattering. We will see below that an exposure 
of $60 \mathrm{~kg}$-year can be realistically achieved with present experiments. This sensitivity is, however, still ten orders of magnitude away from the cross section range shown in figure 8.

In the following, we investigate how realistic the assumptions of no detector energy threshold and only irreducible neutrino background for DM experiments with a LXe target are, taking the example of XENON1T with the so far largest LXe target. Its ultra-low background rate for electron recoils of $82_{-3}^{+5}$ events/(ton·year.keV) at low energies represents the lowest background level in the world for a DM experiment. It is achieved by a strict material selection w.r.t. radio-purity and by fiducialising an inner self-shielded LXe volume. The interaction point is reconstructed by using both the scintillation light (S1) and the charge signal (S2). Unfortunately, demanding an S1 signal restricts the electron recoil energy to more than 2.5 (2.3) keV for XENON10 (XENON100) [81] and $1.3 \mathrm{keV}$ for XENON1T [28]. These energies are significantly larger than the electron binding energy of a few tens of eV. First, we estimate the sensitivity of the XENON1T experiment for the 1 ton-year exposure collected for the spin-independent WIMP nuclear recoil analysis [28], using realistic backgrounds and energy thresholds and the standard mode of fiducialisation with both the S1 and S2 signals. As discussed in ref. [82], the electron recoil background in XENON1T is expected to be flat in the region of interest (ROI) of the WIMP search and dominated by $\beta$-decays of the ${ }^{214} \mathrm{~Pb}$ originating from ${ }^{222} \mathrm{Rn}$ emanations. The largest subdominant background comes from $\beta$-decays of ${ }^{85} \mathrm{Kr}$, whose average natural concentration is reduced through cryogenic distillation down to the sub-ppt level [83]. The energy threshold is defined by a corrected scintillation (cS1) signal between 3 and 70 photoelectrons (PE) and an ionisation (S2) signal threshold of $200 \mathrm{PE}$ [28]. Similarly to the procedure in ref. [84], a conversion function between $\mathrm{S} 1$ in $\mathrm{PE}$ and energy recoil in keV can be derived by using the Noble Element Simulation Technique (NEST) [85]. As further discussed in ref. [86], NEST contains a global analysis of LXe measurements from all available historical data for the mean photon and charge yield, including XENON1T. Thus, a reliable conversion down to the $1 \mathrm{keV}$ energy threshold, where the detection efficiency of XENON1T drops to less than $10 \%$, is available. Using the NEST conversion function, we explore both a conservative detection threshold of $2 \mathrm{keV}$, where a full triggered efficiency is still possible, and for illustration also $1 \mathrm{keV}$, where the detection efficiency is already limited. Using Poisson statistics (cf. table XXII of ref. [87]), we then obtain for each assumed DM mass an average upper limit on the event rate and thus the experimental sensitivity on the reference cross section $\bar{\sigma}_{\chi \mathrm{e}}$ (green and blue lines in figure 9). In principle, the sensitivity can be improved by using the expected annual modulation of a DM signal in an earth-bound detector rotating once per year around the sun and thus possessing an annually modulated velocity w.r.t. to the DM wind. Using this time information, the XENON100 experiment with its electron energy threshold of $2.3 \mathrm{keV}$ became sensitive to DM masses down to $600 \mathrm{MeV}$ with a lowest cross section of $6 \cdot 10^{-35} \mathrm{~cm}^{2}$ for a DM mass of $2 \mathrm{GeV}$ and axial-vector couplings [88] (black line in figure 9).

To really come closer to the ideal sensitivity line, the energy threshold has to be lowered drastically by abandoning the scintillation light requirement S1 and using only the charge signal S2, which has a lower energy threshold. The disadvantage of this method is that fiducialisation is limited, since without S1 the event depth $z$ cannot be accurately estimated, yielding a potentially increased background. Furthermore, there are additional single- 


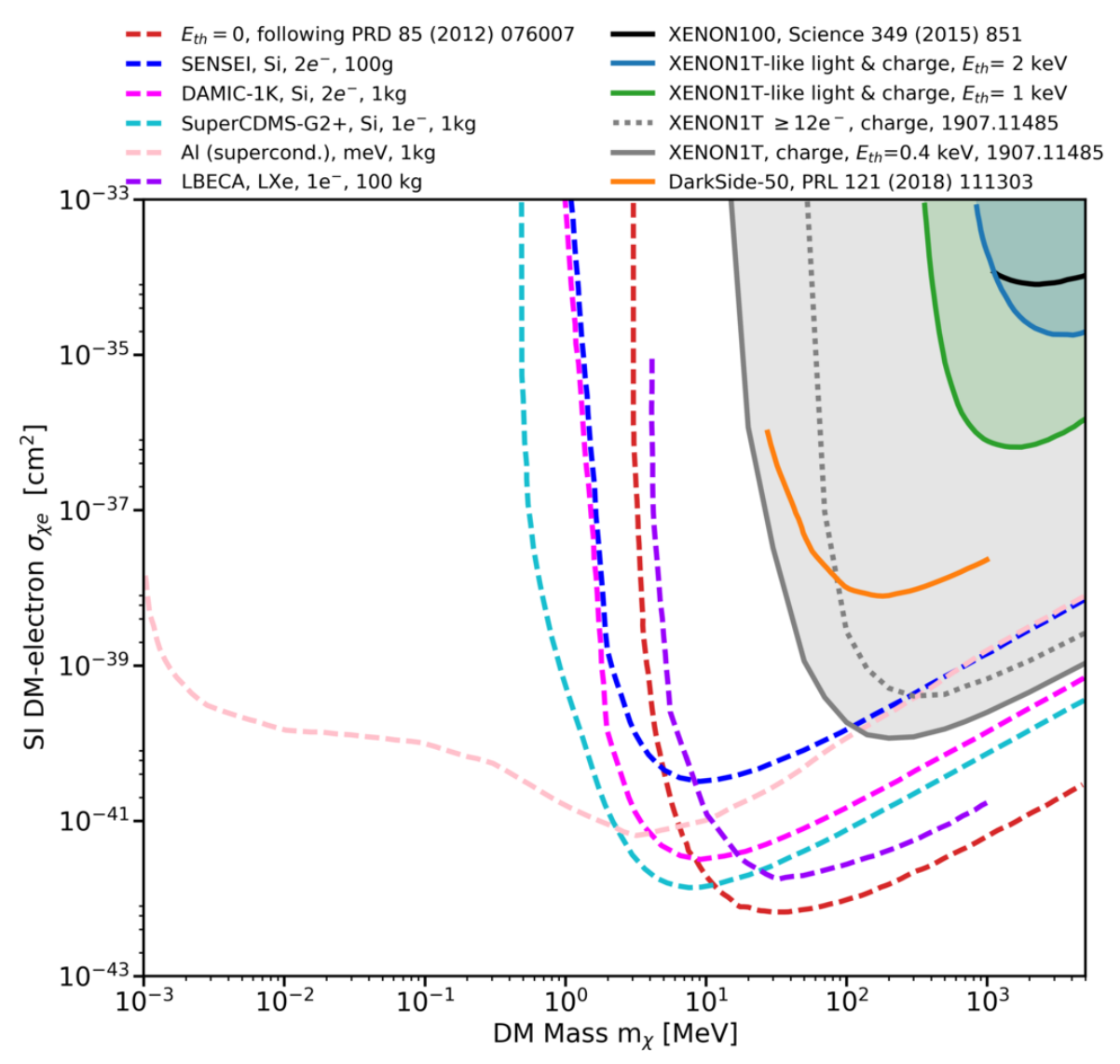

Figure 9. Experimental upper limits and estimated sensitivities for the DM-electron scattering cross section $\bar{\sigma}_{\chi \mathrm{e}}$ in LXe, LAr and other detectors. Shown are the previously estimated sensitivity for a $60 \mathrm{~kg}$-year exposure assuming only irreducible neutrino backgrounds and no detection thresholds (red dotted) [53-56] together with our sensitivity estimates for an assumed electron energy detection threshold of $2 \mathrm{keV}$ (blue) and $1 \mathrm{keV}$ (green), respectively, for a 1 ton-year exposure of XENON1T with realistic assumptions at $95 \%$ confidence level (C.L.) [28]. Also shown are published limits from XENON100 (black) [88] and a charge-only analysis of XENON1T [91] (gray full line), both at $90 \%$ C.L. The gray dotted line is a more conservative version of the latter limit considering that electron recoils below $186 \mathrm{eV}$ (12 produced electrons) are undetectable, as the LXe charge yield $Q_{y}$ has never been measured below these energies [91]. For comparison, we also show the ionisationonly limit in Argon from DarkSide-50 (orange full line) [92] and sensitivity projections (dashed lines) for SENSEI (blue), DAMIC-1K (magenta), Al (supercond.) (pink), SuperCDMS (cyan) [93] and LBECA (purple) [94].

electron backgrounds coming from the photoionisation of impurities of the LXe and the metal components inside the time-projection chamber [89], which are difficult to describe without considering the information provided by the scintillation light. Therefore, a reliable background model becomes difficult. With this method, searches for DM scattering off nuclei with XENON10 with a $1 \mathrm{keV}$ nuclear recoil energy threshold and an exposure of 15 kg-days [90] as well as with XENON100 with a $0.7 \mathrm{keV}$ nuclear recoil energy threshold and an exposure of $30 \mathrm{~kg}$-years [75] have been performed, assuming very conservatively that 
all counts in the search window are potential signal counts. In refs. [53-56] these analyses were re-interpreted in terms of light DM scattering off electrons, and exclusion limits of $3 \cdot 10^{-38} \mathrm{~cm}^{2}$ or just below $10^{-38} \mathrm{~cm}^{2}$ at $100 \mathrm{MeV}$ were obtained.

Very recently XENON1T published a S2-only analysis [91] with an electron energy threshold of $0.4 \mathrm{keV}$. Strict cuts on the data were applied to lower the background drastically down to 1 event/(ton $\cdot$ day $\cdot \mathrm{keV})$, resulting in a remaining effective exposure of 22 ton-days or $60 \mathrm{~kg}$-years. The analysis considered a flat electron recoil background originating mainly from ${ }^{214} \mathrm{~Pb}$ decays, coherent elastic neutrino-nucleon scattering and "cathode events", which were attributed to low energy $\beta$-electrons from the cathode wire grid. This analysis was sensitive to light DM scattering off electrons down to masses of 100 to $20 \mathrm{MeV}$ (gray lines in figure 9).

Comparing the estimated sensitivities and experimental limits plotted in figure 9 to the reference cross section of at most a few times $10^{-52} \mathrm{~cm}^{2}$ in the SLIM model in figure 8, we observe a difference of ten orders of magnitude despite the $\mathrm{MeV}$ to GeV DM mass and large electron couplings. The reason is of course the large suppression by the fourth power in the mass of the charged heavy mediator. Despite the fact that XENON1T has demonstrated unprecedented low background levels, the exclusion reach is still heavily constrained by the detection threshold. Skipping the requirement to detect the scintillation light signal S1 together with the charge signal S2 allowed to lower the energy threshold already by one order of magnitude down to $0.4 \mathrm{keV}$ and the cross section limit by many orders of magnitude. Cryogenic bolometers can have even lower energy thresholds (e.g. CRESST-III [76]), but they suffer from much smaller detector masses. In addition, alternative technologies such as the charge-coupled-device (CCD) experiments SENSEI [95] and DAMIC [96] with $0.1 \mathrm{~kg}$ to $1 \mathrm{~kg}(1 \mathrm{~K})$ target mass, respectively, a low-threshold Generation-2 (G2+) SuperCDMS detector [97] or a superconducting Aluminum cube [98] are currently being explored [93]. The liquid xenon experiment LBECA also aims at a significant background reduction for single or few electron signals [94]. Note that the current limits, e.g. from the first shallow underground run of SENSEI, are still eight orders of magnitude weaker than the projected sensitivity [95]. The projections are therefore shown in figure 9 as dashed lines.

\section{Conclusion}

Motivated by the observations of DM, fundamental scalars and non-zero neutrino masses in Nature, we have studied in this paper right-handed neutrinos with $\mathrm{MeV}$ to $\mathrm{GeV}$ mass, that can explain the DM and generate the active neutrino masses at one loop through their couplings to the SM neutrinos via a dark scalar doublet, mixing with a scalar singlet. When the mass of the lightest neutral scalar is also as light as MeV (i.e. a so-called SLIM), this scotogenic model, as had been observed previously, can in addition solve the cosmological missing satellite, cusp-core and the too-big-to fail problems.

We first scanned the full parameter space with right-handed neutrino DM in the $\mathrm{MeV}$ to $\mathrm{GeV}$ mass range to identify viable models that reproduce the observed SM neutrino mass differences and lead to the correct observed relic density. We found that these models automatically have DM masses above a few $\mathrm{MeV}$ in excellent agreement with bounds from 
big-bang nucleosynthesis. We also observed that they implied sizeable couplings to not only the neutral, but also the charged SM leptons between a few times $10^{-4}$ and a few times $10^{-1}$.

We then turned to lepton flavour violation, which had previously not been analysed, but which occurs naturally in this type of models due to the coupling of the dark sector to both the charged and neutral SM leptons. Specifically, we calculated the expected branching ratio of the process $\mu \rightarrow e \gamma$, which is usually the most sensitive one. This process turned out to be highly constraining, with the current MEG results already eliminating a substantial part of the lepton coupling parameter space. Since the latter is constrained from below from the requirement of non-zero active neutrino masses, the planned MEG upgrade should almost completely verify or exclude this model. The current limits on the processes $\mu \rightarrow 3 e$ and muon to electron conversion in Titanium proved to be less constraining, but the next generation of experiments should be of similar sensitivity.

Since no nuclear recoil signal was expected for this leptophilic DM, we then investigated instead the possibility of an electron signal, which had generally been suggested as a possible way to detect sub-GeV DM. We found that, despite the sizeable coupling of the right-handed neutrino DM to charged leptons, the electron recoil cross section did not exceed a few times $10^{-46} \mathrm{~cm}^{2}$, when all low-energy, collider, cosmological and neutrino mass and mixing constraints were imposed. This result could be traced to the fact that the charged scalar mediator was restricted by LEP searches to be heavier than $98.5 \mathrm{GeV}$ and suppressed the cross section with the fourth power of the mass. Lepton flavour violation constraints limited the electron recoil cross section further down to at most $10^{-52} \mathrm{~cm}^{2}$. We furthermore performed a detailed study of the sensitivity of XENON and other direct detection experiments, in particular to DM induced electron recoil for a 1 ton-year exposure collected with realistic backgrounds and energy thresholds, and compared it to previous theoretical estimates without backgrounds or threshold limitations. Here, we found that cross sections of about $10^{-36} \mathrm{~cm}^{2}$ rather than a few times $10^{-41} \mathrm{~cm}^{2}$ and DM masses of a few $100 \mathrm{MeV}$ rather than a few tens of $\mathrm{MeV}$ were required. Furthermore, we included the recent published S2-only analysis result from XENON1T that features a lower electron energy threshold of $0.4 \mathrm{keV}$ and a simplified, but realistic background model. The cross sections of about $10^{-39} \mathrm{~cm}^{2}$ for DM masses a few tens of $\mathrm{MeV}$ demonstrated several orders of magnitude of improvement in the XENON sensitivity, approaching the optimistic theoretical estimates. This makes it imperative to increase the exposure in the future at a threshold below the keV level, e.g. by exploiting further only the ionisation (S2) signal. There, XENONnT will rival the sensitivities of upcoming or planned dedicated experiments exploring the MeV mass range such as SENSEI, DAMIC-1K, SuperCDMS-G2+ or superconducting Aluminum cubes. We cautioned again that theoretical projections may be overly optimistic, as they were in the case of XENON, since e.g. the proven sensitivity from the first underground run of SENSEI is still eight orders of magnitude below the projection.

We close with two remarks on possible generalisations of this model and future directions of research. Indeed, some of our findings do not depend on the details of the model. In particular, our results should apply more generally to $\mathrm{MeV}$ to $\mathrm{GeV}$ scale neutrino DM and depend neither on the details of the symmetry stabilising the DM nor on the presence of an equally light scalar, as the main feature was the coupling of $\mathrm{MeV}$ neutrino DM to SM 
leptons via the scalar doublet and the LEP limit on the mass of its charged component. In contrast, promoting the U(1) symmetry stabilising the DM to a local symmetry and then studying the phenomenology of its spontaneous breaking at colliders and in cosmology would represent an interesting future direction of research [99-101].

\section{Acknowledgments}

We thank J. Alvey for useful discussions. This work has been supported by the BMBF under contract 05H18PMCC1 and the DFG through the Research Training Group 2149 "Strong and weak interactions - from hadrons to dark matter".

Open Access. This article is distributed under the terms of the Creative Commons Attribution License (CC-BY 4.0), which permits any use, distribution and reproduction in any medium, provided the original author(s) and source are credited.

\section{References}

[1] M. Klasen, M. Pohl and G. Sigl, Indirect and direct search for dark matter, Prog. Part. Nucl. Phys. 85 (2015) 1 [arXiv:1507.03800] [INSPIRE].

[2] PlancK collaboration, Planck 2018 results. VI. Cosmological parameters, arXiv: 1807.06209 [INSPIRE].

[3] G. Jungman, M. Kamionkowski and K. Griest, Supersymmetric dark matter, Phys. Rept. 267 (1996) 195 [hep-ph/9506380] [INSPIRE].

[4] B. Herrmann and M. Klasen, SUSY-QCD corrections to dark matter annihilation in the Higgs funnel, Phys. Rev. D 76 (2007) 117704 [arXiv:0709.0043] [InSPIRE].

[5] B. Herrmann, M. Klasen and K. Kovarik, Neutralino annihilation into massive quarks with SUSY-QCD corrections, Phys. Rev. D 79 (2009) 061701 [arXiv:0901.0481] [InSPIRE].

[6] B. Herrmann, M. Klasen and K. Kovarik, SUSY-QCD effects on neutralino dark matter annihilation beyond scalar or gaugino mass unification, Phys. Rev. D 80 (2009) 085025 [arXiv:0907.0030] [INSPIRE].

[7] J. Harz, B. Herrmann, M. Klasen, K. Kovarik and Q.L. Boulc'h, Neutralino-stop coannihilation into electroweak gauge and Higgs bosons at one loop, Phys. Rev. D 87 (2013) 054031 [arXiv: 1212.5241] [INSPIRE].

[8] B. Herrmann, M. Klasen, K. Kovarik, M. Meinecke and P. Steppeler, One-loop corrections to gaugino (co)annihilation into quarks in the MSSM, Phys. Rev. D 89 (2014) 114012 [arXiv: 1404.2931] [INSPIRE].

[9] J. Harz, B. Herrmann, M. Klasen and K. Kovarik, One-loop corrections to neutralino-stop coannihilation revisited, Phys. Rev. D 91 (2015) 034028 [arXiv:1409.2898] [InSPIRE].

[10] J. Harz, B. Herrmann, M. Klasen, K. Kovař́k and M. Meinecke, SUSY-QCD corrections to stop annihilation into electroweak final states including Coulomb enhancement effects, Phys. Rev. D 91 (2015) 034012 [arXiv:1410.8063] [InSPIRE].

[11] J. Harz, B. Herrmann, M. Klasen, K. Kovarik and P. Steppeler, Theoretical uncertainty of the supersymmetric dark matter relic density from scheme and scale variations, Phys. Rev. D 93 (2016) 114023 [arXiv: 1602.08103] [INSPIRE]. 
[12] S. Schmiemann, J. Harz, B. Herrmann, M. Klasen and K. Kovařík, Squark-pair annihilation into quarks at next-to-leading order, Phys. Rev. D 99 (2019) 095015 [arXiv:1903.10998] [INSPIRE].

[13] N. Baro, F. Boudjema and A. Semenov, Full one-loop corrections to the relic density in the MSSM: a few examples, Phys. Lett. B 660 (2008) 550 [arXiv:0710.1821] [INSPIRE].

[14] N. Baro, F. Boudjema, G. Chalons and S. Hao, Relic density at one-loop with gauge boson pair production, Phys. Rev. D 81 (2010) 015005 [arXiv:0910.3293] [INSPIRE].

[15] F. Boudjema, G. Drieu La Rochelle and S. Kulkarni, One-loop corrections, uncertainties and approximations in neutralino annihilations: examples, Phys. Rev. D 84 (2011) 116001 [arXiv: 1108.4291] [INSPIRE].

[16] F. Boudjema, G. Drieu La Rochelle and A. Mariano, Relic density calculations beyond tree-level, exact calculations versus effective couplings: the ZZ final state, Phys. Rev. D 89 (2014) 115020 [arXiv:1403.7459] [INSPIRE].

[17] M. Beneke, C. Hellmann and P. Ruiz-Femenia, Heavy neutralino relic abundance with Sommerfeld enhancements - a study of pMSSM scenarios, JHEP 03 (2015) 162 [arXiv:1411.6930] [INSPIRE].

[18] M. Beneke, C. Hellmann and P. Ruiz-Femenia, Non-relativistic pair annihilation of nearly mass degenerate neutralinos and charginos III. Computation of the Sommerfeld enhancements, JHEP 05 (2015) 115 [arXiv:1411.6924] [INSPIRE].

[19] M. Beneke et al., Relic density of wino-like dark matter in the MSSM, JHEP 03 (2016) 119 [arXiv: 1601.04718] [INSPIRE].

[20] M. Klasen, K. Kovarik and P. Steppeler, SUSY-QCD corrections for direct detection of neutralino dark matter and correlations with relic density, Phys. Rev. D 94 (2016) 095002 [arXiv: 1607.06396] [INSPIRE].

[21] J. Debove, B. Fuks and M. Klasen, Transverse-momentum resummation for gaugino-pair production at hadron colliders, Phys. Lett. B 688 (2010) 208 [arXiv:0907.1105] [INSPIRE].

[22] J. Debove, B. Fuks and M. Klasen, Threshold resummation for gaugino pair production at hadron colliders, Nucl. Phys. B 842 (2011) 51 [arXiv: 1005.2909] [INSPIRE].

[23] J. Debove, B. Fuks and M. Klasen, Joint resummation for gaugino pair production at hadron colliders, Nucl. Phys. B 849 (2011) 64 [arXiv:1102.4422] [INSPIRE].

[24] B. Fuks, M. Klasen, D.R. Lamprea and M. Rothering, Gaugino production in proton-proton collisions at a center-of-mass energy of 8 TeV, JHEP 10 (2012) 081 [arXiv:1207.2159] [INSPIRE].

[25] B. Fuks, M. Klasen, D.R. Lamprea and M. Rothering, Precision predictions for electroweak superpartner production at hadron colliders with resummino, Eur. Phys. J. C 73 (2013) 2480 [arXiv: 1304.0790] [INSPIRE].

[26] B. Fuks, M. Klasen and M. Rothering, Soft gluon resummation for associated gluino-gaugino production at the LHC, JHEP 07 (2016) 053 [arXiv: 1604.01023] [INSPIRE].

[27] J. Fiaschi and M. Klasen, Neutralino-chargino pair production at $N L O+N L L$ with resummation-improved parton density functions for LHC run II, Phys. Rev. D 98 (2018) 055014 [arXiv: 1805.11322] [INSPIRE].

[28] XENON collaboration, Dark matter search results from a one ton-year exposure of XENON1 T, Phys. Rev. Lett. 121 (2018) 111302 [arXiv:1805.12562] [INSPIRE]. 
[29] V. Dutta, S.L. Williams and F. Alonso, Searches for electroweak production of SUSY at $C M S$, talks at EPS HEP, Ghent, Belgium (2019).

[30] ATLAS collaboration, Observation of a new particle in the search for the Standard Model Higgs boson with the ATLAS detector at the LHC, Phys. Lett. B 716 (2012) 1 [arXiv:1207.7214] [INSPIRE].

[31] CMS collaboration, Observation of a new boson at a mass of $125 \mathrm{GeV}$ with the CMS experiment at the LHC, Phys. Lett. B 716 (2012) 30 [arXiv:1207.7235] [INSPIRE].

[32] L. Lopez Honorez, E. Nezri, J.F. Oliver and M.H.G. Tytgat, The inert doublet model: an archetype for dark matter, JCAP 02 (2007) 028 [hep-ph/0612275] [INSPIRE].

[33] M. Klasen, C.E. Yaguna and J.D. Ruiz-Alvarez, Electroweak corrections to the direct detection cross section of inert Higgs dark matter, Phys. Rev. D 87 (2013) 075025 [arXiv: 1302.1657] [INSPIRE].

[34] A. Goudelis, B. Herrmann and O. Stål, Dark matter in the inert doublet model after the discovery of a Higgs-like boson at the LHC, JHEP 09 (2013) 106 [arXiv:1303.3010] [INSPIRE].

[35] Particle Data Group collaboration, Review of particle physics, Phys. Rev. D 98 (2018) 030001 [INSPIRE].

[36] E. Ma, Verifiable radiative seesaw mechanism of neutrino mass and dark matter, Phys. Rev. D 73 (2006) 077301 [hep-ph/0601225] [INSPIRE].

[37] D. Restrepo, O. Zapata and C.E. Yaguna, Models with radiative neutrino masses and viable dark matter candidates, JHEP 11 (2013) 011 [arXiv:1308.3655] [INSPIRE].

[38] M. Klasen, C.E. Yaguna, J.D. Ruiz-Alvarez, D. Restrepo and O. Zapata, Scalar dark matter and fermion coannihilations in the radiative seesaw model, JCAP 04 (2013) 044 [arXiv: 1302.5298] [INSPIRE].

[39] S. Esch, M. Klasen, D.R. Lamprea and C.E. Yaguna, Lepton flavor violation and scalar dark matter in a radiative model of neutrino masses, Eur. Phys. J. C 78 (2018) 88 [arXiv: 1602.05137] [INSPIRE].

[40] S. Esch, M. Klasen and C.E. Yaguna, A singlet doublet dark matter model with radiative neutrino masses, JHEP 10 (2018) 055 [arXiv:1804.03384] [INSPIRE].

[41] J. Fiaschi, M. Klasen and S. May, Singlet-doublet fermion and triplet scalar dark matter with radiative neutrino masses, JHEP 05 (2019) 015 [arXiv: 1812.11133] [INSPIRE].

[42] V. Springel et al., Simulating the joint evolution of quasars, galaxies and their large-scale distribution, Nature 435 (2005) 629 [astro-ph/0504097] [INSPIRE].

[43] M. Drewes et al., A white paper on keV sterile neutrino dark matter, JCAP 01 (2017) 025 [arXiv: 1602.04816] [INSPIRE].

[44] C. Bœhm, Y. Farzan, T. Hambye, S. Palomares-Ruiz and S. Pascoli, Is it possible to explain neutrino masses with scalar dark matter?, Phys. Rev. D 77 (2008) 043516 [hep-ph/0612228] [INSPIRE].

[45] Y. Farzan, A minimal model linking two great mysteries: neutrino mass and dark matter, Phys. Rev. D 80 (2009) 073009 [arXiv: 0908.3729] [INSPIRE].

[46] Y. Farzan, Strategies to link tiny neutrino masses with huge missing mass of the universe, Int. J. Mod. Phys. A 26 (2011) 2461 [arXiv:1106.2948] [InSPIRE].

[47] C. Boehm, D. Hooper, J. Silk, M. Casse and J. Paul, MeV dark matter: has it been detected?, Phys. Rev. Lett. 92 (2004) 101301 [astro-ph/0309686] [INSPIRE]. 
[48] A. Arhrib, C. Bohm, E. Ma and T.-C. Yuan, Radiative model of neutrino mass with neutrino interacting MeV dark matter, JCAP 04 (2016) 049 [arXiv: 1512.08796] [INSPIRE].

[49] J. Kubo, E. Ma and D. Suematsu, Cold dark matter, radiative neutrino mass, $\mu \rightarrow$ e $\gamma$ and neutrinoless double beta decay, Phys. Lett. B 642 (2006) 18 [hep-ph/0604114] [INSPIRE].

[50] J. Kopp, V. Niro, T. Schwetz and J. Zupan, DAMA/LIBRA and leptonically interacting dark matter, Phys. Rev. D 80 (2009) 083502 [arXiv:0907.3159] [INSPIRE].

[51] D. Schmidt, T. Schwetz and T. Toma, Direct detection of leptophilic dark matter in a model with radiative neutrino masses, Phys. Rev. D 85 (2012) 073009 [arXiv:1201.0906] [INSPIRE].

[52] P.-H. Gu and X.-G. He, Electrophilic dark matter with dark photon: from DAMPE to direct detection, Phys. Lett. B 778 (2018) 292 [arXiv:1711.11000] [INSPIRE].

[53] R. Essig, J. Mardon and T. Volansky, Direct detection of sub-GeV dark matter, Phys. Rev. D 85 (2012) 076007 [arXiv: 1108.5383] [InSPIRE].

[54] R. Essig, A. Manalaysay, J. Mardon, P. Sorensen and T. Volansky, First direct detection limits on sub-GeV dark matter from XENON10, Phys. Rev. Lett. 109 (2012) 021301 [arXiv:1206.2644] [INSPIRE].

[55] R. Essig, M. Fernandez-Serra, J. Mardon, A. Soto, T. Volansky and T.-T. Yu, Direct detection of sub-GeV dark matter with semiconductor targets, JHEP 05 (2016) 046 [arXiv: 1509.01598] [INSPIRE].

[56] R. Essig, T. Volansky and T.-T. Yu, New constraints and prospects for sub-GeV dark matter scattering off electrons in xenon, Phys. Rev. D 96 (2017) 043017 [arXiv: 1703. 00910] [INSPIRE].

[57] S.K. Lee, M. Lisanti, S. Mishra-Sharma and B.R. Safdi, Modulation effects in dark matter-electron scattering experiments, Phys. Rev. D 92 (2015) 083517 [arXiv: 1508.07361] [INSPIRE].

[58] ATLAS collaboration, Combination of searches for invisible Higgs boson decays with the ATLAS experiment, Phys. Rev. Lett. 122 (2019) 231801 [arXiv:1904.05105] [INSPIRE].

[59] CMS collaboration, Search for invisible decays of a Higgs boson produced through vector boson fusion in proton-proton collisions at $\sqrt{s}=13$ TeV, Phys. Lett. B 793 (2019) 520 [arXiv: 1809.05937] [INSPIRE].

[60] OPAL collaboration, Search for stable and longlived massive charged particles in $e^{+} e^{-}$ collisions at $\sqrt{s}=130 \mathrm{GeV}$ to $209 \mathrm{GeV}$, Phys. Lett. B 572 (2003) 8 [hep-ex/0305031] [INSPIRE].

[61] CMS collaboration, Combined measurements of Higgs boson couplings in proton-proton collisions at $\sqrt{s}=13 \mathrm{TeV}$, Eur. Phys. J. C 79 (2019) 421 [arXiv:1809.10733] [INSPIRE].

[62] C. Bøhm, P. Fayet and R. Schaeffer, Constraining dark matter candidates from structure formation, Phys. Lett. B 518 (2001) 8 [astro-ph/0012504] [INSPIRE].

[63] C. Bœhm and R. Schaeffer, Constraints on dark matter interactions from structure formation: damping lengths, Astron. Astrophys. 438 (2005) 419 [astro-ph/0410591] [INSPIRE].

[64] J.A. Casas and A. Ibarra, Oscillating neutrinos and $\mu \rightarrow e, \gamma$, Nucl. Phys. B 618 (2001) 171 [hep-ph/0103065] [INSPIRE].

[65] S. May, Minimal dark matter models with radiative neutrino masses: from Lagrangians to observables, M.Sc. thesis, University of Münster, Münster, Germany (2018). 
[66] F. Staub, SARAH 4: a tool for (not only SUSY) model builders, Comput. Phys. Commun. 185 (2014) 1773 [arXiv: 1309.7223] [INSPIRE].

[67] W. Porod and F. Staub, SPheno 3.1: extensions including flavour, CP-phases and models beyond the MSSM, Comput. Phys. Commun. 183 (2012) 2458 [arXiv:1104.1573] [INSPIRE].

[68] D. Barducci et al., Collider limits on new physics within MicrOMEGAs_4.3, Comput. Phys. Commun. 222 (2018) 327 [arXiv:1606.03834] [INSPIRE].

[69] MEG collaboration, Search for the lepton flavour violating decay $\mu^{+} \rightarrow e^{+} \gamma$ with the full dataset of the MEG experiment, Eur. Phys. J. C 76 (2016) 434 [arXiv:1605.05081] [INSPIRE].

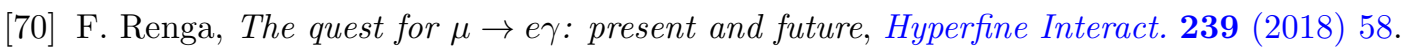

[71] SINDRUM collaboration, Search for the decay $\mu^{+} \rightarrow e^{+} e^{+} e^{-}$, Nucl. Phys. B 299 (1988) 1 [INSPIRE].

[72] SINDRUM II collaboration, Test of lepton flavor conservation in $\mu \rightarrow e$ conversion on titanium, Phys. Lett. B 317 (1993) 631 [INSPIRE].

[73] A. Blondel et al., Research proposal for an experiment to search for the decay $\mu \rightarrow$ eee, arXiv:1301.6113 [INSPIRE].

[74] A. Sato, R\&D of muon storage ring PRISM-FFAG to improve a sensitivity of $\mu$-e conv. experiment beyond $B R \sim 10^{-17}$, PoS (NUFACT08) 105 (2009).

[75] XENON collaboration, Low-mass dark matter search using ionization signals in XENON100, Phys. Rev. D 94 (2016) 092001 [Erratum ibid. D 95 (2017) 059901] [arXiv: 1605. 06262] [INSPIRE].

[76] CRESST collaboration, First results from the CRESST-III low-mass dark matter program, arXiv: 1904.00498 [INSPIRE].

[77] J.I. Read, The local dark matter density, J. Phys. G 41 (2014) 063101 [arXiv:1404.1938] [INSPIRE].

[78] C. Bunge, J. Barrientos and A. Bunge, Roothaan-Hartree-Fock ground-state atomic wave functions: slater-type orbital expansions and expectation values for $z=2-54$, Atom. Data Nucl. Data Tabl. 53 (1993) 113.

[79] M.C. Smith et al., The RAVE survey: constraining the local galactic escape speed, Mon. Not. Roy. Astron. Soc. 379 (2007) 755 [astro-ph/0611671] [INSPIRE].

[80] P.F. Depta, M. Hufnagel, K. Schmidt-Hoberg and S. Wild, BBN constraints on the annihilation of MeV-scale dark matter, JCAP 04 (2019) 029 [arXiv:1901.06944] [INSPIRE].

[81] L. Baudis et al., Response of liquid xenon to Compton electrons down to $1.5 \mathrm{keV}$, Phys. Rev. D 87 (2013) 115015 [arXiv: 1303.6891] [INSPIRE].

[82] XENON collaboration, Physics reach of the XENON1T dark matter experiment, JCAP 04 (2016) 027 [arXiv: 1512.07501] [INSPIRE].

[83] XENON collaboration, Removing krypton from xenon by cryogenic distillation to the ppq level, Eur. Phys. J. C 77 (2017) 275 [arXiv:1612.04284] [INSPIRE].

[84] XENON100 collaboration, First axion results from the XENON100 experiment, Phys. Rev. D 90 (2014) 062009 [Erratum ibid. D 95 (2017) 029904] [arXiv: 1404.1455] [INSPIRE]. 
[85] M. Szydagis et al., NEST: a comprehensive model for scintillation yield in liquid xenon, 2011 JINST 6 P10002 [arXiv:1106.1613] [INSPIRE].

[86] XENON collaboration, XENON1T dark matter data analysis: signal and background models and statistical inference, Phys. Rev. D 99 (2019) 112009 [arXiv:1902.11297] [INSPIRE].

[87] G.J. Feldman and R.D. Cousins, A unified approach to the classical statistical analysis of small signals, Phys. Rev. D 57 (1998) 3873 [physics/9711021] [INSPIRE].

[88] XENON100 collaboration, Exclusion of leptophilic dark matter models using XENON100 electronic recoil data, Science 349 (2015) 851 [arXiv: 1507.07747] [INSPIRE].

[89] XENON100 collaboration, Observation and applications of single-electron charge signals in the XENON100 experiment, J. Phys. G 41 (2014) 035201 [arXiv:1311.1088] [inSPIRE].

[90] XENON10 collaboration, A search for light dark matter in XENON10 data, Phys. Rev. Lett. 107 (2011) 051301 [Erratum ibid. 110 (2013) 249901] [arXiv:1104.3088] [INSPIRE].

[91] XENON collaboration, Light dark matter search with ionization signals in XENON1T, arXiv: 1907.11485 [INSPIRE].

[92] DARKSide collaboration, Constraints on sub-GeV dark-matter-electron scattering from the DarkSide-50 experiment, Phys. Rev. Lett. 121 (2018) 111303 [arXiv:1802.06998] [INSPIRE].

[93] M. Battaglieri et al., U.S. cosmic visions: new ideas in dark matter 2017. Community report, in U.S. cosmic visions: new ideas in dark matter, College Park, MD, U.S.A., 23-25 March 2017 [arXiv: 1707.04591] [INSPIRE].

[94] K. Ni, LBECA: a Low Background Electron Counting Apparatus for sub-GeV dark matter detection, talk given at TAUP, Toyoma, Japan (2019).

[95] SENSEI collaboration, SENSEI: direct-detection constraints on sub-GeV dark matter from a shallow underground run using a prototype skipper-CCD, Phys. Rev. Lett. 122 (2019) 161801 [arXiv: 1901.10478] [INSPIRE].

[96] DAMIC collaboration, First direct-detection constraints on eV-scale hidden-photon dark matter with DAMIC at SNOLAB, Phys. Rev. Lett. 118 (2017) 141803 [arXiv:1611.03066] [INSPIRE].

[97] SuPERCDMS collaboration, New results from the search for low-mass weakly interacting massive particles with the CDMS low ionization threshold experiment, Phys. Rev. Lett. 116 (2016) 071301 [arXiv: 1509.02448] [inSPIRE].

[98] Y. Hochberg, M. Pyle, Y. Zhao and K.M. Zurek, Detecting superlight dark matter with Fermi-degenerate materials, JHEP 08 (2016) 057 [arXiv: 1512.04533] [INSPIRE].

[99] M. Klasen, F. Lyonnet and F.S. Queiroz, $N L O+N L L$ collider bounds, Dirac fermion and scalar dark matter in the B-L model, Eur. Phys. J. C 77 (2017) 348 [arXiv:1607.06468] [INSPIRE].

[100] D.A. Camargo, M.D. Campos, T.B. de Melo and F.S. Queiroz, A two Higgs doublet model for dark matter and neutrino masses, Phys. Lett. B 795 (2019) 319 [arXiv:1901.05476] [INSPIRE].

[101] D.A. Camargo, M. Klasen and S. Zeinstra, Discovering heavy U(1)-gauged Higgs bosons at the HL-LHC, arXiv:1903.02572 [INSPIRE]. 